\title{
Effect of spanwise domain size on direct numerical simulations of airfoil noise during flow separation and stall
}

\author{
Jacob M. Turnera) and Jae Wook Kim \\ Institute of Sound 83 Vibration Research, University of Southampton \\ Southampton, SO17 1BJ, United Kingdom
}

(Dated: 3 June 2020)

It is well established that a large spanwise domain size is required for accurate numerical simulations of flow past an airfoil in stall. A number of numerical experiments support this conclusion with regard to aerodynamic and turbulence statistics. However, very little has been reported concerning the effect of span length on aeroacoustic results. In this paper a detailed investigation is carried out into the influence of spanwise domain length on the prediction of airfoil stall noise when spanwise periodic boundary conditions are applied. The study is based on direct numerical simulations of a NACA0012 airfoil at $R e_{\infty}=50,000$ and $M_{\infty}=0.4$ at near- and full-stall conditions. There are three main findings in the paper. Firstly, the far-field acoustics are found to be highly sensitive to the choice of spanwise domain length. In the full-stall case, a span length equal to $20 \%$ of the airfoil chord over-predicts the radiated noise by more than $10 \mathrm{~dB}$ at low-to-medium frequencies relative to a case with one chord length in span. Discrepancies are found to occur for acoustic wavelengths shorter than the spanwise domain size. Under near-stall conditions the changes caused by the small spanwise domain are noticeably milder. Secondly, the lower noise predictions from the large span simulation at low frequencies is attributed to spanwise breakup of large scale flow structures, and reduced spanwise coherence near the trailing edge. Thirdly, a more destructive source phase relationship is observed with large span for medium frequencies, which was inhibited by the periodic forcing in the small span case.

\section{INTRODUCTION}

Airfoil self-noise can be separated into five categories: turbulent boundary layer scattering at the trailing edge (TBL-TE), laminar shedding, tip noise, TE bluntness shedding, and noise from flow separation ${ }^{1}$. For airfoils at low angles of attack TBL-TE is typically considered the dominant source of noise. It has received great attention including significant efforts to understand its generation mechanisms, and how to effectively reduce it. Noise from flow separation and stall however, has received relatively little attention. This is despite airfoils being regularly operated at high angles in real engineering applications where strong adverse pressure gradients are likely. Such a scenario is particularly probable when airfoils are subjected to unsteady inflow conditions, for example wind shear encountered by wind turbines. It has been suggested that noise from flow separation could reasonably exceed low angle TBL-TE by more than $10 \mathrm{~dB}^{2}$.

Stall noise has been documented by experimental studies 1 17, where the key emerging feature is a high level of low frequency sound. Various airfoil profiles have been tested by Moreau et al.$^{3}$ at Reynolds number $R e_{\infty}=150,000$ and for Mach numbers $M_{\infty}<0.1$. They identified a primarily dipolar noise signature, with a distinction between light and deep stall regimes. The latter was shown to exhibit additional peaks, which were speculated to result from shear layer instabilities or coherent shedding. These findings were later reiterated by Laratro et al ${ }^{[4}$ at $R e_{\infty}=64,000$ and 96, 000. Laratro et al. also showed that although the noise level in stall was comparable with different airfoil profiles, it varies at stall onset proportionally to the severity of lift changes (sharper stall producing more noise).

Presently there is no clear consensus for the noise generation mechanisms in stall. Paterson et al ${ }^{[5}$ found a correlation between the far-field noise and the wall pressure over the last $30 \%$ of the aerofoil chord, therefore suggesting that the acoustic source region is not restricted to the TE (as it is for low angles of attack). This result suggests that the same noise reduction techniques (e.g. TE serrations) developed for TE noise may be less effective under separated flow conditions. Three possible mechanisms for the noise generation have been suggested in a recent paper by Lacagnina et al ${ }^{6}$ for near stall conditions. They theorize that shear layer instabilities, flapping, and shear layer coherent shedding could be a source of unsteady pressure on the wall, which convects towards, and is scattered by the TE.

There is a requirement for high fidelity numerical simulations to better understand noise generation in the stall regime. In particular, to identify the near-field acoustic sources from the hydrodynamic field. Despite this, to date

a) Electronic mail: j.m.turner@soton.ac.uk 
the stall regime represents a significant challenge for numerical approaches because of computational cost limitations. Traditionally, airfoil simulations save cost by considering very small spans and relying on the implementation of spanwise periodic boundary conditions to capture 3D effects. This approach is usually reasonable at small angles of attack where spanwise length scales are also small. However, for high angles much larger spans may be required. There is evidence to suggest span lengths of approximately one chord length may be necessary, even for accurate prediction of mean aerodynamic quantities ${ }^{8}$. However, relatively little has been reported regarding the effect of span length on acoustic predictions. The aim of the current study is to investigate how numerical predictions of the far-field noise are influenced by the choice of spanwise domain size. This is necessary before any meaningful conclusions can be drawn from future in-depth numerical studies on the noise mechanisms in the stall regime.

In this paper we consider direct numerical simulations (DNS) of a NACA0012 airfoil at two angles of attack: $\alpha=10^{\circ}$ (near stall) and $\alpha=15^{\circ}$ (full stall). Three spanwise domain lengths are tested, 20\%, 60\%, and 100\%, of the airfoil chord length. The work continues from an earlier study based on small span solutions ${ }^{9}$. The main finding of the paper is that the simulation based on a small spanwise domain length over-predicts the radiated noise at low-medium frequencies by more than $10 \mathrm{~dB}$ relative to the large spanwise domain case. For low frequencies this is attributed to an increase of spanwise coherence for flow structures at the TE and in the wake. Additionally, at medium frequencies, an increased constructive source phase relationship is observed over the airfoil surface.

The remainder of the paper is organized as follows: Section II describes the current numerical methodology, including numerical schemes, meshing, and far-field sound extrapolation. In section III A qualitative differences in the flow field produced by different span simulations are discussed, as well as changes in aerodynamic quantities. In section IIIB the far-field acoustic data is presented for the two angles of attack with different span lengths including times signals, spectra, and directivity patterns. Next in section IIIC the differences in the dipole source characteristics are shown, which provide an explanation for the changes to the far-field noise. This includes two-dimensional contours of wall pressure spectra and phase information. Finally in section IV concluding remarks are given.

\section{PROBLEM DESCRIPTION}

In this paper we consider a NACA0012 airfoil at free-stream Reynolds number $R e_{\infty}=50,000$ and Mach number $M_{\infty}=0.4$. Two angles of attack are considered $\alpha=10^{\circ}$ (near stall) and $\alpha=15^{\circ}$ (deep stall), with three spanwise domain lengths: $L_{z}=0.2 L_{c}$ (small), $0.6 L_{c}$ (medium), and $L_{c}$ (large), where $L_{c}$ is one chord length. Compressible flow data is obtained by using a direct numerical simulation (DNS), whereas the acoustic field is predicted with a Ffowcs-Williams and Hawkings (FW-H) code.

\section{A. Governing equations and numerical methods}

The compressible 3-D Navier-Stokes equations written in a conservative form for a generalized coordinate system are:

$$
\frac{\partial}{\partial t}\left(\frac{\boldsymbol{Q}}{J}\right)+\frac{\partial}{\partial \xi_{i}}\left(\frac{\boldsymbol{E}_{j}-R e_{\infty}^{-1} M_{\infty} \boldsymbol{F}_{j}}{J} \frac{\partial \xi_{i}}{\partial x_{j}}\right)=-\frac{a_{\infty}}{L_{c}} \frac{\boldsymbol{S}}{J},
$$

where the indices $i=1,2,3$ and $j=1,2,3$ denote the three dimensions; $a_{\infty}$ is the ambient speed of sound, and $\boldsymbol{S}$ is an optional source term utilized in a sponge layer. The vectors of the conservative variables, inviscid and viscous fluxes are given by

$$
\left.\begin{array}{c}
\boldsymbol{Q}=\left[\rho, \rho u, \rho v, \rho w, \rho e_{\mathrm{t}}\right]^{T}, \\
\boldsymbol{E}_{j}=\left[\rho u_{j},\left(\rho u u_{j}+\delta_{1 j} p\right),\left(\rho v u_{j}+\delta_{2 j} p\right),\left(\rho w u_{j}+\delta_{3 j} p\right),\left(\rho e_{\mathrm{t}}+p\right) u_{j}\right]^{T}, \\
\boldsymbol{F}_{j}=\left[0, \tau_{1 j}, \tau_{2 j}, \tau_{3 j}, u_{i} \tau_{j i}+q_{j}\right]^{T},
\end{array}\right\}
$$

with the stress tensor and heat flux vector written as

$$
\tau_{i j}=\mu\left(\frac{\partial u_{i}}{\partial x_{j}}+\frac{\partial u_{j}}{\partial x_{i}}-\frac{2}{3} \delta_{i j} \frac{\partial u_{i}}{\partial x_{i}}\right), \quad q_{j}=\frac{\mu}{(\gamma-1) \operatorname{Pr}} \frac{\partial T}{\partial x_{j}},
$$

where $\xi_{i}=\{\xi, \eta, \zeta\}$ are the generalized body fitted coordinates (aligned in the streamwise, cross-stream and lateral directions, respectively), $x_{j}=\{x, y, z\}$ are the Cartesian coordinates, $\delta_{i j}$ is the Kronecker delta, $u_{j}=\{u, v, w\}$, $e_{\mathrm{t}}=p /[(\gamma-1) \rho]+u_{j} u_{j} / 2$ and $\gamma=1.4$ for air. The local dynamic viscosity $\mu$ is calculated by using Sutherland's law $\frac{10}{10}$. The Jacobian determinant of the coordinate transformation (from Cartesian to the generalized) is given by 
$J^{-1}=\mid \partial(x, y, z) / \partial(\xi, \eta, \zeta)[11$. The governing equations are non-dimensionalized. Length scales are normalized by the airfoil chord length $L_{c}$, velocities by the ambient speed of sound $a_{\infty}$, time scales by $L_{c} / a_{\infty}$ and pressure by $\rho_{\infty} a_{\infty}^{2}$. Temperature, density and dynamic viscosity are normalized by their respective ambient values: $T_{\infty}, \rho_{\infty}$ and $\mu_{\infty}$.

At longitudinal and vertical far-field boundaries general characteristic-based non-reflecting boundary conditions $(\text { GCBCs })^{12}$ are implemented in order to prevent any reflections from outgoing waves. Additionally, disturbances near the boundaries are damped towards the required mean flow condition by a sponge zone implemented via the source term $\boldsymbol{S}$ in Eq. 1 13|14 This term is non-zero within the sponge layer only. Any acoustic waves are attenuated and absorbed in the sponge layer to prevent numerical reflections. In the spanwise direction periodic conditions are applied as indicated earlier, while on the airfoil surface a no-slip wall boundary conditions is used ${ }^{15}$. For each simulation the angle of attack is set by adjusting the inflow velocity through a moving frame technique. The velocity is ramped up from zero to $\left(u_{\infty}, v_{\infty}, w_{\infty}\right)=(|\boldsymbol{u}| \cos (\alpha),|\boldsymbol{u}| \sin (\alpha), 0)$ over a period of $t^{*}=t a_{\infty} / L_{c}=5$ non-dimensional time units.

The governing equations given above are solved by using high-order accurate numerical methods specifically developed for aeroacoustic simulations on structured grids. The flux derivatives in space are calculated based on fourth-order pentadiagonal compact finite difference schemes with seven-point stencils $\$$. Explicit time advancing of the numerical solution is carried out by using the classical fourth-order Runge-Kutta scheme with the CFL number of 0.95. Numerical stability is maintained by implementing sixth-order pentadiagonal compact filters for which the cut-off wavenumber (normalized by the grid spacing) is set to $\pi$ at the boundaries and $0.85 \pi$ in the interior regions 17 .

The computation is parallelized via domain decomposition and message passing interface (MPI) routines. The compact finite difference schemes and filters are implicit in space and require an inversion of a set of pentadiagonal coefficient matrices. This calculation requires a precise and efficient technique for parallel simulations in order to avoid numerical artifacts that may appear at the subdomain (processor core) boundaries. A quasi-disjoint matrix systems $\frac{18}{18}$ approach offering super-linear scalability is used for parallelisation in the present paper. Calculations are carried out on the UK national supercomputer ARCHER as well as the IRIDIS5 cluster at the University of Southampton. The grid is distributed onto 1200 processor cores for the smallest case, up to 6720 for the largest.

\section{B. Domain, geometry and meshing}

The computational domain is a rectangular cuboid with a sharp trailing edge NACA0012 airfoil at the centre shown in figure 1 $(a)$. The longitudinal and vertical boundaries of the domain are surrounded by the sponge layer through which the flow is (gently) forced to the mean flow condition. The entire computational domain; the inner region (physical domain) where meaningful simulation data are obtained; and, the sponge layer zone are defined as

$$
\left.\begin{array}{c}
\mathcal{D}_{\infty}=\left\{\boldsymbol{x} \mid x / L_{c} \in[-9,9], y / L_{c} \in[-9,9], z \in\left[-L_{z} / 2, L_{z} / 2\right]\right\}, \\
\mathcal{D}_{\text {physical }}=\left\{\boldsymbol{x} \mid x / L_{c} \in[-7,7], y / L_{c} \in[-7,7], z \in\left[-L_{z} / 2, L_{z} / 2\right]\right\}, \\
\mathcal{D}_{\text {sponge }}=\mathcal{D}_{\infty}-\mathcal{D}_{\text {physical }},
\end{array}\right\}
$$

where $L_{c}$ denotes the airfoil chord length. The airfoil's leading and trailing edges are located at $x / L_{c}=-1 / 2$ and $1 / 2$, respectively.

The domain is decomposed into six blocks which make up an H-topology grid system. The grid is stretched in two stages from the airfoil surface to the far-field. A relatively fine grid is maintained for the first two chord lengths above the airfoil which approximately corresponds to the wake height at the outlet boundary for $\alpha=15^{\circ}$ angle of attack. The grid is then stretched more rapidly in the far-field region. For the same purpose a large number of points are maintained up to two chords downstream of the TE to properly capture the wake in the streamwise direction. The smallest cells are clustered at the airfoil leading and trailing edges, while a uniform spacing is used in the spanwise direction (which is preserved for each spanwise domain size). The number of grid cells used is $\left(N_{x}, N_{y}\right)=(1200,1120)$ in two-dimensions ( $x y$-plane), with $N_{z}=66,196$, and 326 respectively for $L_{z} / L_{c}=0.2,0.6$, and 1.0 cases.

The grid is shown in figure 1 $(b)$ for the airfoil wake, $(c)$ near the airfoil, $(d)$ on the airfoil surface. The grid is asymmetric in the vertical direction with additional refinement on the suction side of the airfoil to capture the separated shear layer away from the wall and boundary layer transition to turbulence. In figure $1(b)$ the grid lines are colored by velocity magnitude (for $\alpha=15^{\circ}$ ) which demonstrates how the grid is refined in the wake region. Figure $2(a)$ and $(b)$ shows the surface mesh sizes on the suction side in wall units for $\alpha=10^{\circ}$ and $\alpha=15^{\circ}$ respectively. For $\alpha=10^{\circ}$ over the majority of the wall $2 \leq \Delta s^{+} \leq 10.5, \Delta n^{+}<1$, and $3 \leq \Delta z^{+} \leq 11.5$, where $\Delta s$ and $\Delta n$ represent the wall tangential and normal spacings respectively. For $\alpha=15^{\circ}, 1 \leq \Delta s^{+} \leq 5, \Delta n^{+}<1$, and $2 \leq \Delta z^{+} \leq 8$. The current spacings are below the recommended values for DNS suggested by Georgiadis et al.199.

Since the current simulations consider separated flows, the wall mesh sizes alone are not an adequate predictor for grid resolution. It is also important to verify that the mesh sizes away from the wall are sufficient to capture the separated shear layer and wake. In order to achieve this the grid is compared to the Kolmogorov microscale 
(a)
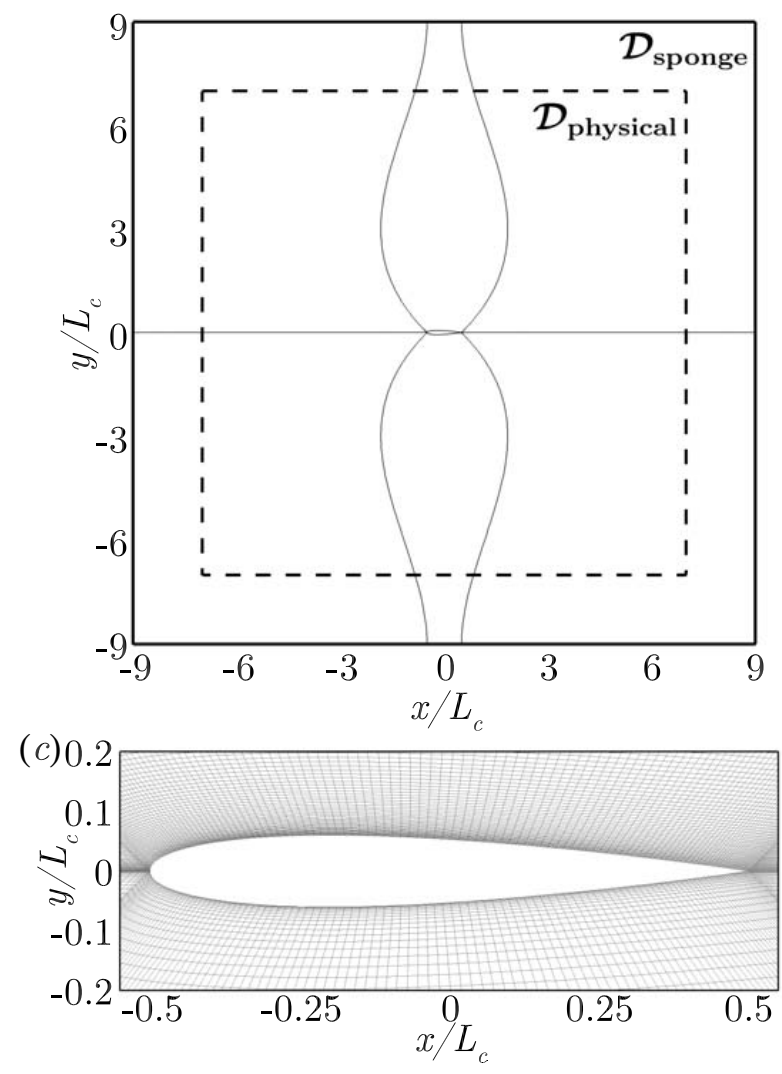

(b)

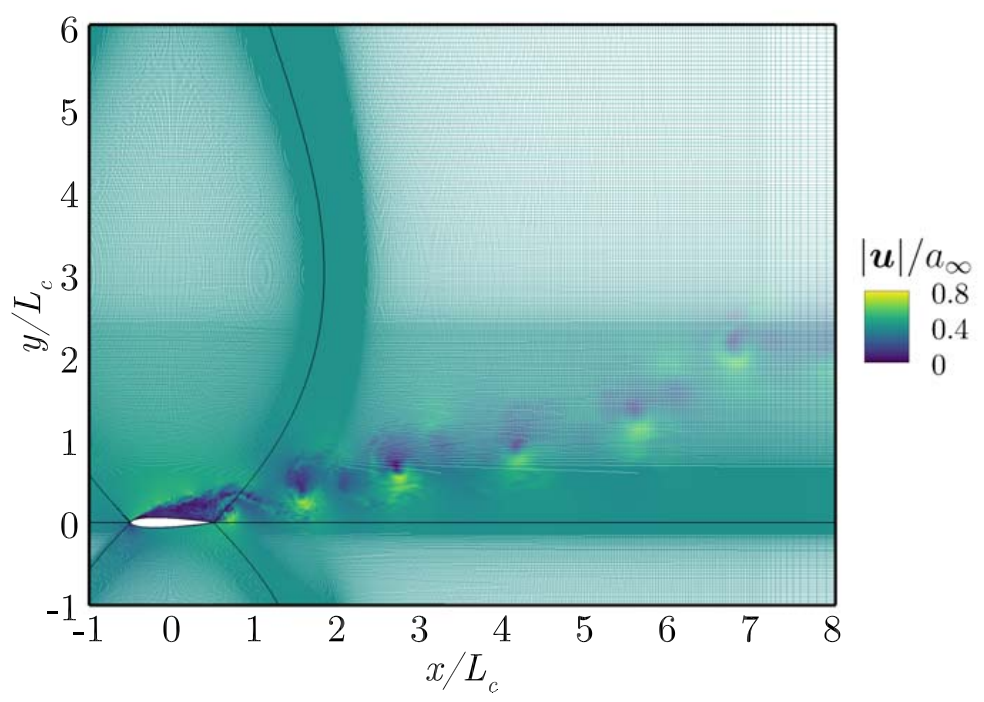

(d)

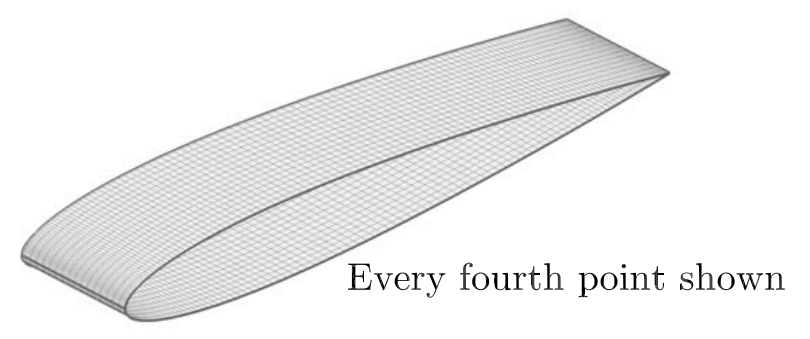

FIG. 1. Computational domain and mesh used in the numerical setup. (a) Computational domain including physical and sponge zones. (b) Zoomed out side view of the grid showing the airfoil wake. Grid lines colored by velocity magnitude $|\boldsymbol{u}| / a_{\infty}$. (c) Airfoil side view close up (every fourth point shown in each direction). (d) Surface mesh shown for $20 \%$ of the span (every fourth point shown in each direction).

$\eta=\left(\nu^{3} / \epsilon\right)^{1 / 4}$, where $\epsilon$ is the turbulence dissipation rate $\epsilon=2 \nu \overline{\mathcal{S}_{i j} \mathcal{S}_{i j}}$, and $\mathcal{S}_{i j}$ is the rate of strain tensor. A ballpark target value of $\Delta x \approx 5 \eta$ is obtained by considering the requirements for grid generated free turbulence simulations suggested by Laizet et al. 20 . This was shown to obtain $<5 \%$ error relative to experiment predictions for first and second order moments. Figure $2(c)$ shows a contour plot of the ratio between cube root of cell volume, and the Kolmogorov microscale on the airfoil suction side in the $x y$-plane. The volume is calculated via the Jacobian, and results are shown for $\alpha=10^{\circ}$. The highest value is approximately $5.5 \eta$, with the majority of the turbulent region satisfying $J^{1 / 3}<4 \eta$.

\section{Validation of numerical simulations}

In order to verify the current grid settings are sufficient the current DNS is compared to an ILES (implicit large eddy simulation) solution. The ILES grid is obtained by reducing the number of points in the streamwise, lateral and spanwise directions by a factor of two, as well as doubling the minimum spacings on the wall. This results in a grid with a total of $54.77 \mathrm{M}$ points compared to $438.1 \mathrm{M}$ points for the DNS. The time and spanwise averaged pressure $\left(C_{p}\right)$ and skin friction $\left(C_{f}\right)$ coefficients are compared in figure $3(a)$ and $(b)$ for the largest span $\left(L_{z}=L_{c}\right)$. For both variables there is a close agreement, therefore demonstrating a reasonable level of grid convergence.

In figure $4(a)$ and $(b)$ the time averaged coefficients of lift $\left(C_{L}\right)$ and $\operatorname{drag}\left(C_{D}\right)$ obtained by the large spanwise domain DNS are compared to various experimental values 2123 in the Reynolds number range $10^{4} \leq R e_{\infty} \leq 10^{5}$. The current solution falls within the range of expected values suggested by the experimental data. Relative to the experiment by Ohtake et al. ${ }^{22}\left(R e_{\infty}=50,000\right)$ there is a $6.6 \%$ and $1.8 \%$ over-prediction for $C_{L}$ and $C_{D}$, whereas compared to Tsuchiya et al ${ }^{23}\left(R e_{\infty}=47,000\right)$ the lift and drag are underestimated by $3.0 \%$ and $5.5 \%$ respectively. 
(a)

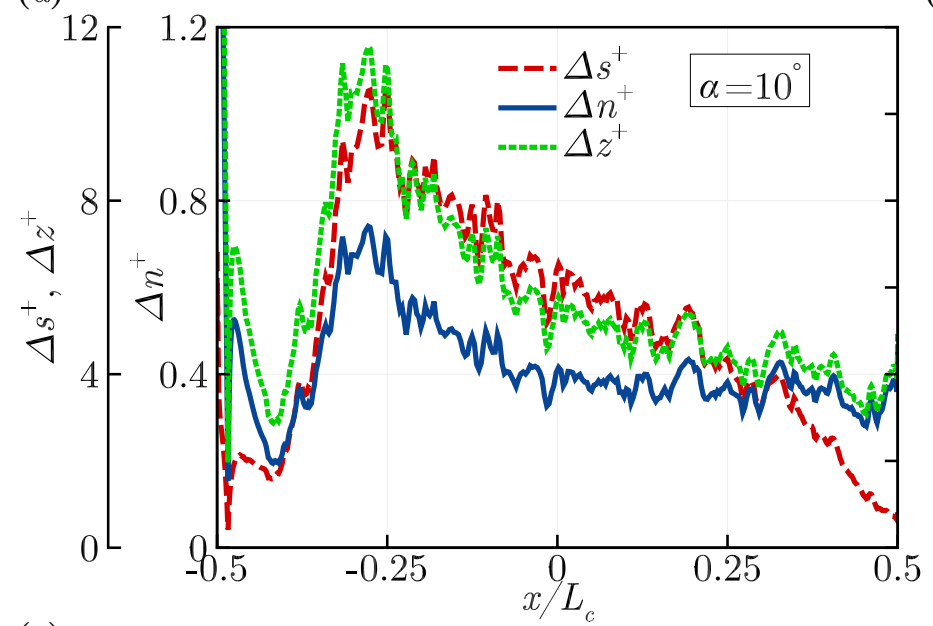

(b)

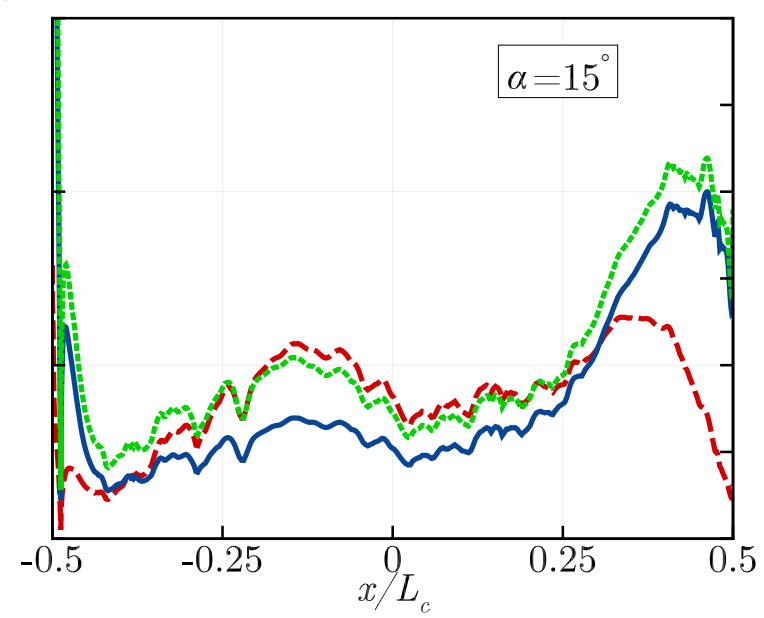

$(c)$

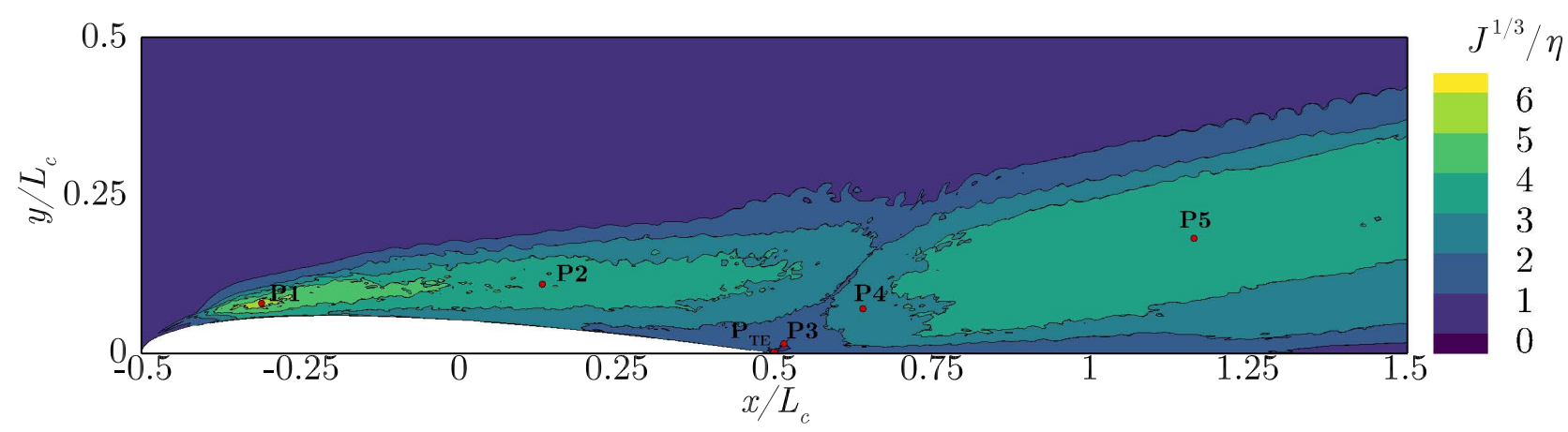

FIG. 2. Instantaneous grid sizes in wall units over the airfoil surface averaged in span at $t a_{\infty} / L_{c}=200:(a) \alpha=10^{\circ} ;(b)$ $\alpha=15^{\circ} . \Delta s$ and $\Delta n$ represent the wall tangential fitted and wall normal spacings respectively. (c) Cube root of cell volume compared to the Kolmogorov microscale in the $x y$-plane (averaged in span) for $\alpha=10^{\circ}$.

(a)

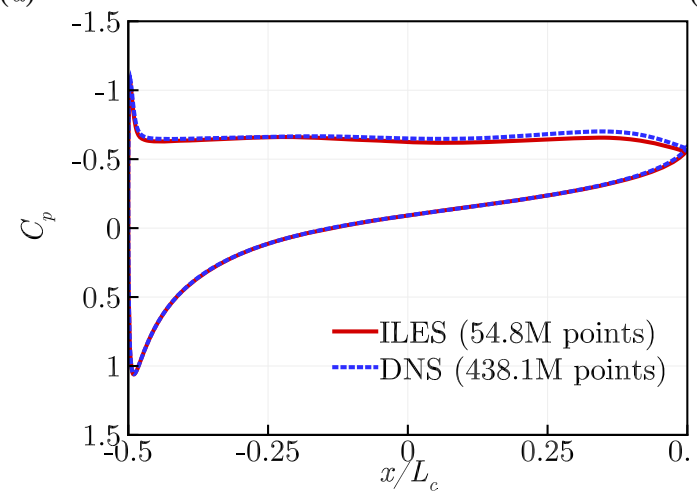

(b)

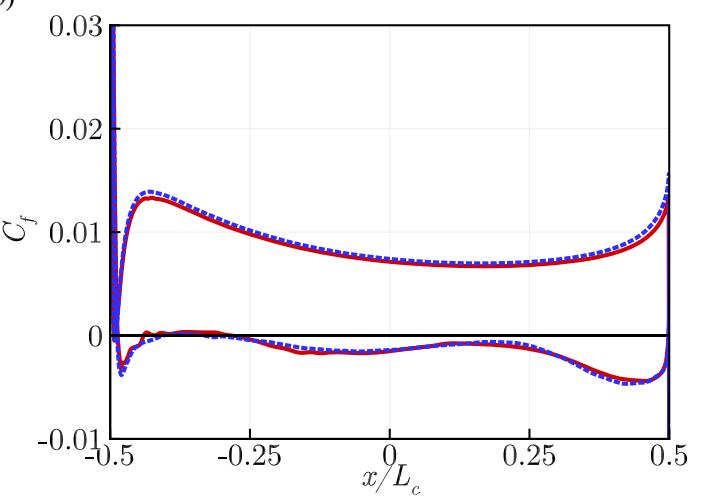

FIG. 3. Comparison of $C_{p}(a)$ and $C_{f}(b)$ obtained by the current DNS for $\alpha=15^{\circ}$ and $L_{z}=L_{c}$ with an ILES (implicit large eddy simulation) solution coarsened by a factor of 2 in each direction.

\section{Far-field extrapolation approach}

In order to calculate the noise in the acoustic far-field a time domain solution of the Ffowcs-Williams and Hawkings equation is implemented based on the Farassat 1A formulation $\stackrel{2425}{ }$, simplified for the current problem:

$$
4 \pi p_{a}(\boldsymbol{x}, t)=\int\left[\frac{\dot{p} \cos (\Phi)}{a_{\infty} r\left(1-M_{r}\right)^{2}}+\frac{p\left(\cos (\Phi)-M_{i} n_{i}\right)}{r^{2}\left(1-M_{r}\right)^{2}}+\frac{\left(M_{r}-M^{2}\right) p \cos (\Phi)}{r^{2}\left(1-M_{r}\right)^{3}}+\frac{\rho_{\infty} a_{\infty} u_{i} n_{i}\left(M_{r}-M^{2}\right)}{r^{2}\left(1-M_{r}\right)^{3}}\right]_{r e t} \mathrm{~d} S
$$


(a)

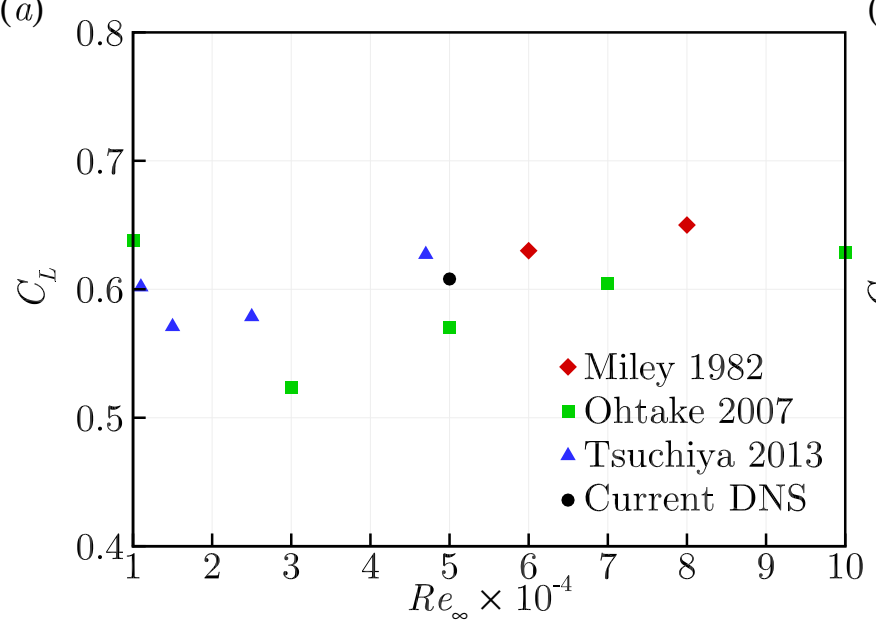

(b)

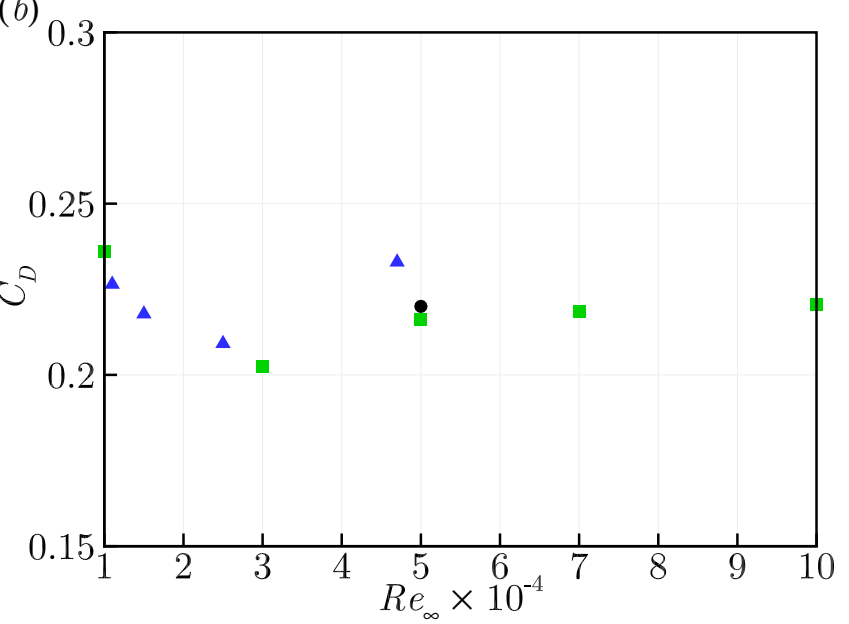

FIG. 4. Comparison of $C_{L}(a)$ and $C_{D}(b)$ values obtained by the large spanwise length simulation $\left(L_{z}=L_{c}\right)$ at $\alpha=15^{\circ}$ with the experimental data of Miley ${ }^{21}$, Ohtake et al. ${ }^{[2]}$ and Tsuchiya et al. ${ }^{23}$, at various Reynolds numbers.

where subscript ret indicates variables are analyzed at the retarded time $\tau=t-r / a_{\infty}$, dotted variables indicate the time derivative, and $r$ is the effective acoustic distance calculated from the Garrick triangle ${ }^{26}$ extended for twodimensional mean flow velocity:

$$
r=\frac{M_{x} d_{x}+M_{y} d_{y}+\sqrt{\left(M_{x} d_{x}+M_{y} d_{y}\right)^{2}+\left(1-M_{x}^{2}-M_{y}^{2}\right)\left[d_{x}^{2}+d_{y}^{2}+d_{z}^{2}\right]}}{1-M_{x}^{2}-M_{y}^{2}},
$$

where $\left(M_{x}, M_{y}\right)=\left(M_{\infty} \cos (\alpha), M_{\infty} \sin (\alpha)\right)$ and $\left(d_{x}, d_{y}, d_{z}\right)=\left(x_{o}-x_{s}, y_{o}-y_{s}, z_{o}-z_{s}\right)$, with subscript $o$ and $s$ representing observer and source respectively. Additionally, $M_{r}=\boldsymbol{M} \cdot \hat{\boldsymbol{r}}$, where $\boldsymbol{r}$ is the effective radiation vector, and ` represents a unit length. The local radiation angle $\Phi$, is determined by $\Phi=\operatorname{acos}(\boldsymbol{n} \cdot \hat{\boldsymbol{r}})$, where $\boldsymbol{n}$ is the surface normal. The above integration is performed on the airfoil surface, and therefore mainly considers the dipole noise sources (although scattering of quadrupole sound by the wall might also contribute). Impenetrable surface integration greatly reduces the cost of the calculation requiring only the pressure as an input variable.

Since the current simulations include a periodic boundary condition in the spanwise direction, integrating only the computational domain is not sufficient to obtain the far-field noise. In order to do this the periodic boundary condition must be replicated by performing the surface integral iteratively on domains shifted $n L_{z}$ either side of the airfoil (see figure $5(a)$ ). This procedure also allows a fair comparison to be made between the three spanwise domain size simulations. Validation of the current FW-H implementation is also included in figure $5(b)$, based on a previous work of the authors where direct far-field measurements were obtained for airfoil-vortex interaction simulations 27 . The previous work considered a flat plate airfoil in an inviscid subsonic flow with a spanwise domain length of $L_{z}=(2 / 15) L_{c}$. The far-field observer was positioned five chord lengths directly above the airfoil mid-point $\left(x_{o}, y_{o}, z_{o}\right)=\left(0,5 L_{c}, 0\right)$. In this paper a total spanwise length of $3 L_{c}$ is used throughout, or in other words fifteen repeated domains for the smallest span, five for the medium span, and three for the largest span.

\section{E. Definition of variables for statistical analysis}

Data processing and analysis are carried out upon the completion of each simulation. The main property required in this study is the power spectral density (PSD) function of pressure fluctuations on the airfoil surface and at the far-field observer location. The far-field (acoustic) pressure is defined as:

$$
p_{a}(\boldsymbol{x}, t)=p(\boldsymbol{x}, t)-\bar{p}(\boldsymbol{x}),
$$

where $\bar{p}(\boldsymbol{x})$ is the time averaged pressure. We also define the vertical wall pressure jump as:

$$
\Delta p_{w}(x, z, t)=p_{w}\left(x, y_{u}, z, t\right)-p_{w}\left(x, y_{l}, z, t\right)
$$


$(a)$

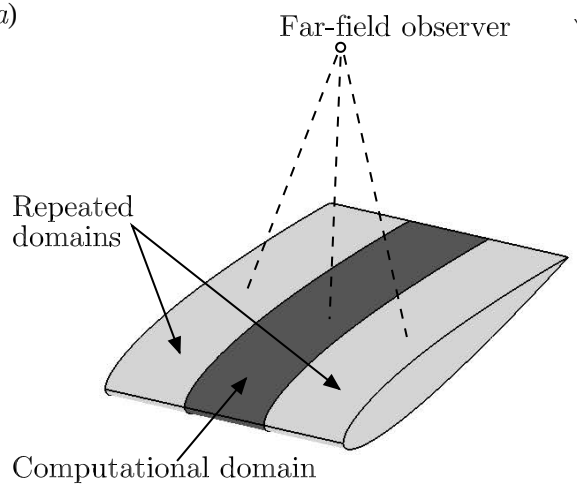

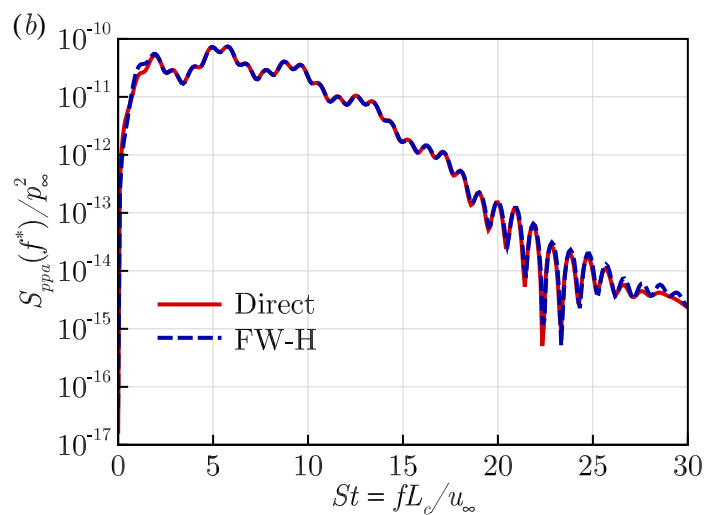

FIG. 5. (a) Schematic diagram of the periodic boundary condition implemented in the FW-H solver which is required in order to compare different span lengths. (b) Validation of current $\mathrm{FW}-\mathrm{H}$ implementation. Comparison is made with direct noise computations of sound generated by an airfoil interacting with a synthetic vortex 27 .

where $p_{w}$ is obtained via Eq.(7) on the airfoil surface, and the subscripts $u$ and $l$ indicate the upper and lower sides of the airfoil. Following the definitions used in 28 , the PSD functions of the pressure fluctuations (based on frequency and one-sided) are then calculated by:

$$
\begin{gathered}
S_{p p a}(\boldsymbol{x}, f)=\lim _{T \rightarrow \infty} \frac{P_{a}(\boldsymbol{x}, f, T) P_{a}^{*}(\boldsymbol{x}, f, T)}{T}, \\
S_{p p w}(\boldsymbol{x}, f)=\lim _{T \rightarrow \infty} \frac{\Delta P_{w}(\boldsymbol{x}, f, T) \Delta P_{w}^{*}(\boldsymbol{x}, f, T)}{T},
\end{gathered}
$$

where $P_{a}$ and $\Delta P_{w}$ are an approximate Fourier transform of $p_{a}$ and $\Delta p_{w}$, respectively, based on the following definitions:

$$
\begin{gathered}
P_{a}(\boldsymbol{x}, f, T)=\int_{-T}^{T} p_{a}(\boldsymbol{x}, t) e^{2 \pi i f t} \mathrm{~d} t, \\
\Delta P_{w}(x, z, f, T)=\int_{-T}^{T} \Delta p_{w}(x, z, t) e^{2 \pi i f t} \mathrm{~d} t,
\end{gathered}
$$

and, '*' denotes a complex conjugate. The same definitions are used to calculate the energy spectrum of velocity fluctuations later in this paper.

\section{INFLUENCE OF AIRFOIL SPAN ON NUMERICAL PREDICTIONS}

The numerical simulations are run with an adaptive time step of approximately $\Delta t^{*}=1 \times 10^{-4}$ for 200 nondimensional time units. Data acquisition is carried out in the last 20 time units with a sample rate of $\Delta t^{*}=0.02$ providing 1000 flow snapshots. The flow field obtained for the two angles of attack tested is shown by iso-surfaces of $Q$-criterion, colored by velocity magnitude in figure 6 $(a)$ and $(b)$ for $L_{z}=L_{c}$. At the current Reynolds number $(50,000)$ the airfoil undergoes leading edge (LE) separation in the form of a laminar separation bubble, which as the angle of attack is increased eventually bursts leading to a full stall scenario. At $\alpha=10^{\circ}$ the airfoil is at the onset of stall, the separation bubble is positioned close to the LE, and is followed by turbulent transition and reattachment further downstream. At $\alpha=15^{\circ}$ the boundary layer is fully separated. The flow is mainly characterized by a separated shear layer near the LE, which develops into large scale vortex shedding near the trailing edge (TE). The energy spectra based on streamwise, vertical and lateral velocity fluctuations is shown in figure $7(a)$ and $(b)$ for the two angles of attack with large spanwise domain length. Four probe points are selected for each case moving from the LE to downstream, their location is shown in figure 2(c). Probes P1, P2, P4 and P5 are shown for $\alpha=10^{\circ}$, while $\mathrm{P} 2-\mathrm{P} 5$ are shown for $\alpha=15^{\circ}$. P3 replaces the probe $\mathrm{P} 1$ for $\alpha=15^{\circ}$ since the flow is not fully developed close to the LE. The spectra obtained by each probe is shifted vertically by $30 \mathrm{~dB}$ for comparison purposes. The classical $-5 / 3$ 

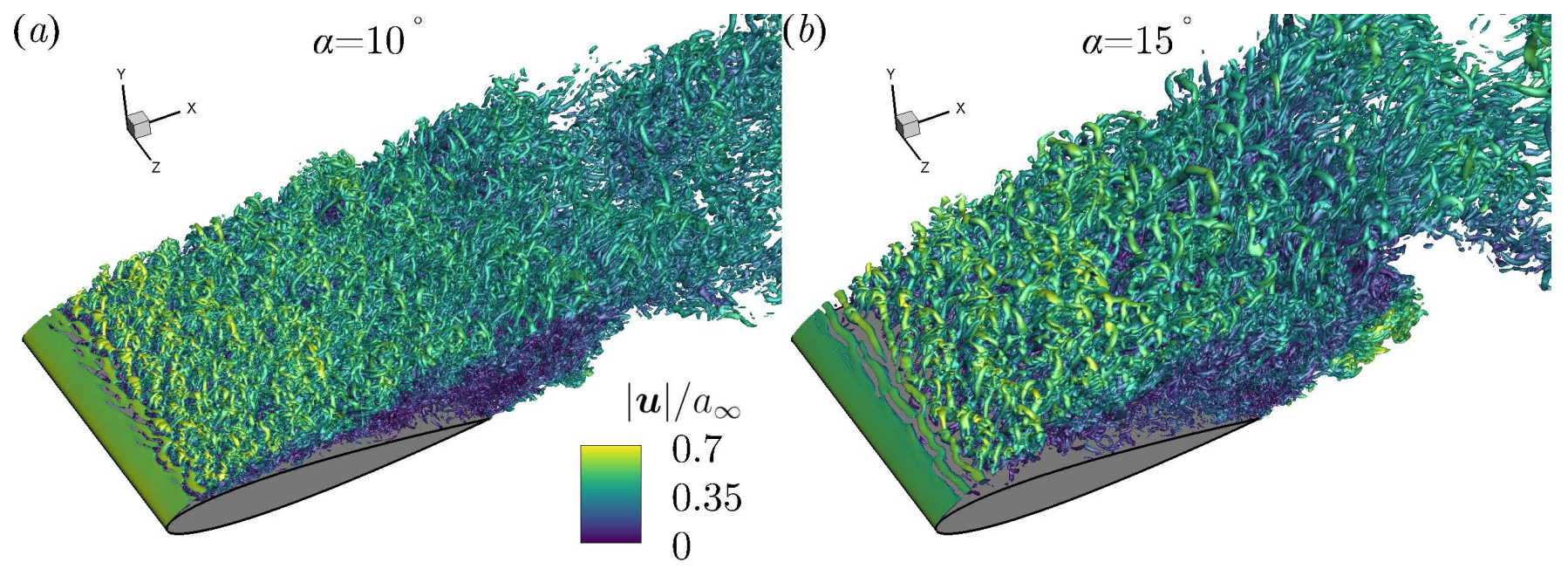

FIG. 6. Flow field visualisation for large span $L_{z}=L_{c}$. (a) Iso-surfaces of $Q$-criterion $(Q=30)$ colored by velocity magnitude $|\boldsymbol{u}| / a_{\infty}$ at $\alpha=10^{\circ} ;(b) 15^{\circ}$.

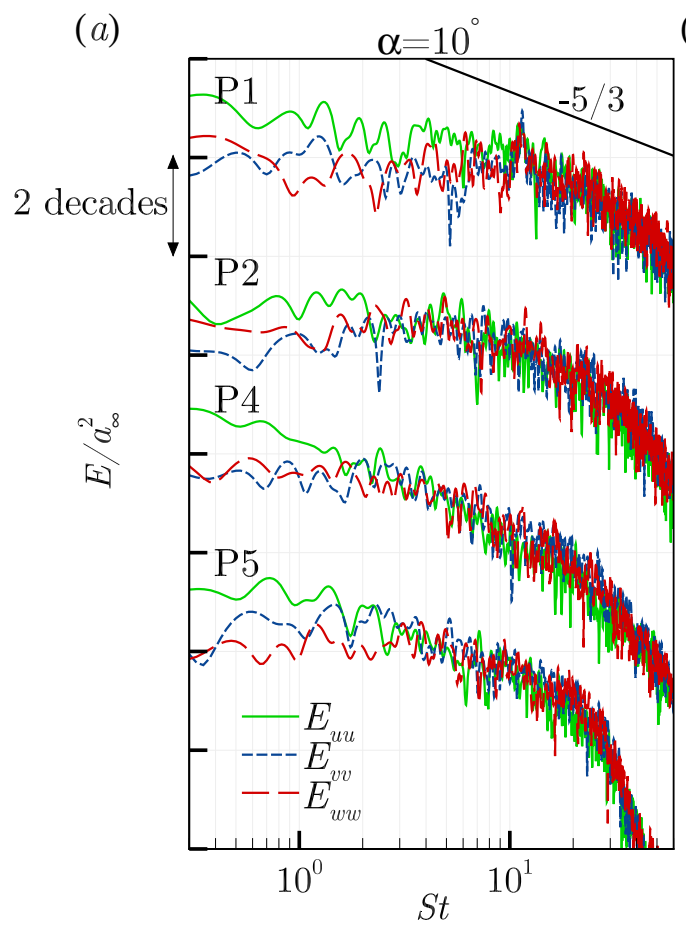

(b)

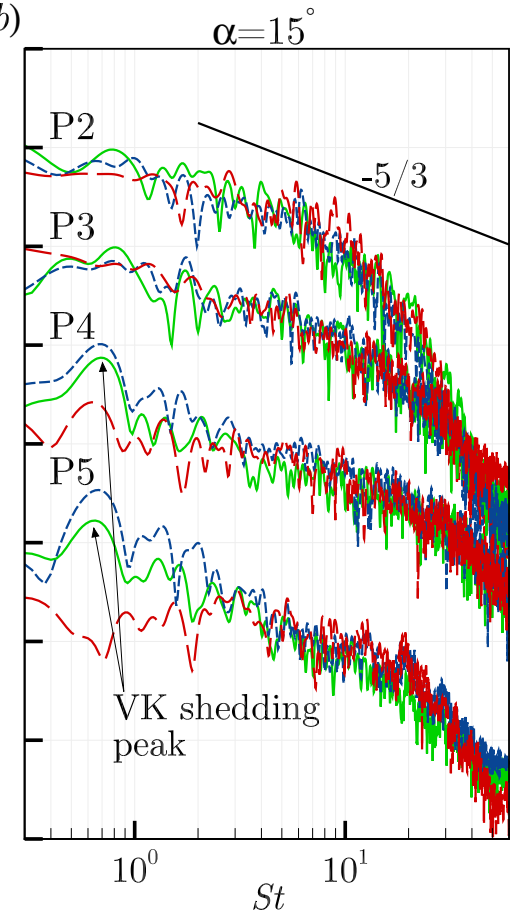

FIG. 7. Energy spectra of streamwise $E_{u u}$, vertical $E_{v v}$ and lateral $E_{w w}$ velocity fluctuations vs Strouhal number $\left(S t=f L_{c} / u_{\infty}\right)$ based on the large span simulation $L_{z}=L_{c}$. (a) $\alpha=10^{\circ}$ and $(b) \alpha=15^{\circ}$. Probe points P1-P5 are shown in figure $2(c)$.

slope for the inertial sub-range is clearly obtained for probes P4 and P5 where the turbulence is fully developed in the airfoil wake. A low frequency peak associated with von Kármán vortex shedding is also clearly visible for $\alpha=15^{\circ}$, primarily in the streamwise and vertical velocity fluctuations, but also to a lesser extent in spanwise fluctuations at $\mathrm{P} 4$. Another interesting observations is made at $\mathrm{P} 1$ for $\alpha=10^{\circ}$ which contains a tone at $S t=12.0$. At $\alpha=10^{\circ}, \mathrm{P} 1$ is situated in the separated region, which could indicate this tone is linked to instabilities in the shear layer such as Kelvin-Helmholtz vortices. 
(a)

$(c)$

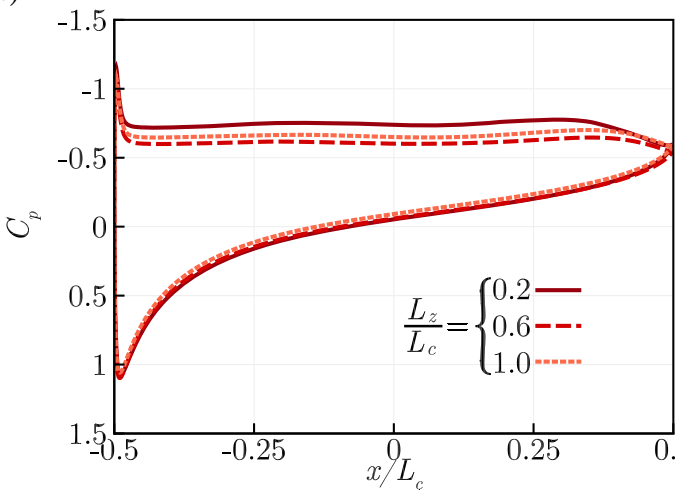

(b)

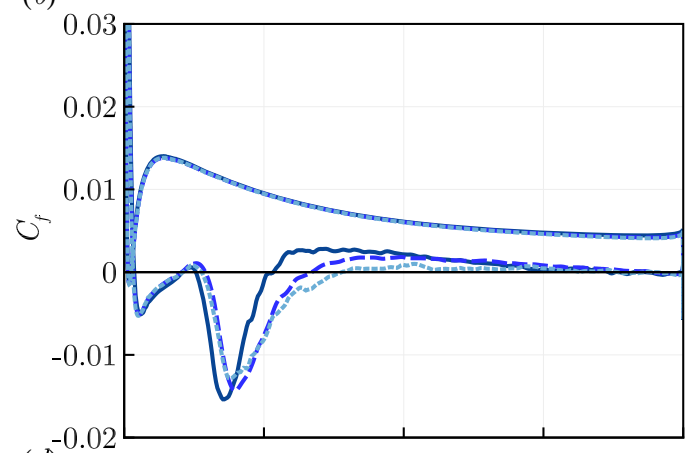

(d)

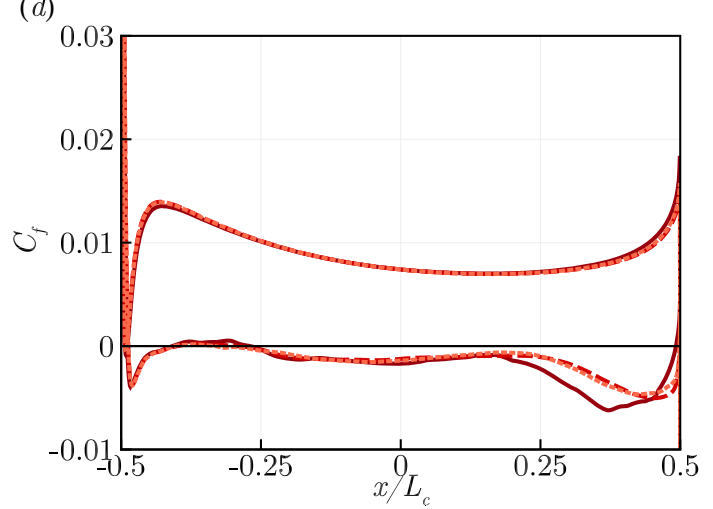

FIG. 8. Time averaged pressure $C_{p}$ and skin friction $C_{f}$ coefficients for $(a \& b) \alpha=10^{\circ}$, and $(c \& d) \alpha=15^{\circ}$, with $L_{z}=0.2 L_{c}$, $L_{z}=0.6 L_{c}$, and $L_{z}=L_{c}$.

\begin{tabular}{ccccc} 
& \multicolumn{2}{c}{$\alpha=10^{\circ}$} & \multicolumn{2}{c}{$\alpha=15^{\circ}$} \\
\hline$L_{z}$ & $C_{L}$ & $C_{D}$ & $C_{L}$ & $C_{D}$ \\
\hline $0.2 L_{c}$ & 0.773 & 0.0741 & 0.715 & 0.246 \\
$0.6 L_{c}$ & 0.834 & 0.0870 & 0.593 & 0.216 \\
$1.0 L_{c}$ & 0.862 & 0.0927 & 0.608 & 0.220 \\
\hline
\end{tabular}

TABLE I. Lift and drag coefficient obtained by the three airfoil span lengths for $\alpha=10^{\circ}$ and $15^{\circ}$.

\section{A. Aerodynamics and flow structure differences}

The spanwise and time averaged pressure $\left(C_{p}\right)$ and skin friction $\left(C_{f}\right)$ coefficients are compared for the three airfoil spanwise domain lengths and two angles of attack in figure $8(a)-(d)$. For both angles of attack the medium and large span length airfoils obtain a reasonably similar result for both $C_{p}$ and $C_{f}$, although the medium span slightly under-predicts the length of the separation bubble compared to the larger span length case. The small span on the other hand shows overall poorer agreement with the other two cases. At $\alpha=10^{\circ}$ the suction side pressure is underpredicted slightly during the bubble, and over-predicted during the turbulent region relative to the large spanwise domain solution. The flow also transitions and reattaches earlier, around $10 \%$ of the chord further upstream. At $\alpha=15^{\circ}$, the pressure on the upper side of the airfoil is consistently lower. This results in a $18 \%$ increase of lift and $12 \%$ increase of drag relative to the large span as shown in table I. This is consistent with the findings of George and Lele $^{\sqrt[8]{8}}$, who showed an overestimate of lift for a small span simulation in the stall regime. The small span airfoil also shows some disagreement in the recirculation region close to the TE, which is both stronger and broader than the larger span cases.

Differences in both the flow structure and radiated sound field are demonstrated in figure 9 for $L_{z}=0.2 L_{c}$ and $L_{z}=L_{c}$. The flow is visualized by $Q$-criterion iso-surfaces, colored by Lamb vector magnitude $(|\boldsymbol{\omega} \times \boldsymbol{u}|)$, while the near-field sound is shown by velocity divergence contours $(\nabla \cdot \boldsymbol{u})$ in the $x y$-plane. The small span is repeated five times for comparison purposes. It is clear how the small span solution limits the scale of 3D structures in the separated shear layer, as well as delaying the breakup of von Kármán structures in the wake. Regarding the sound field, the small spanwise domain solution appears to over-predicts the level of generated sound compared to the larger spanwise domain, particularly emerging from the separated shear layer and underside of the wake. 
$(a)$

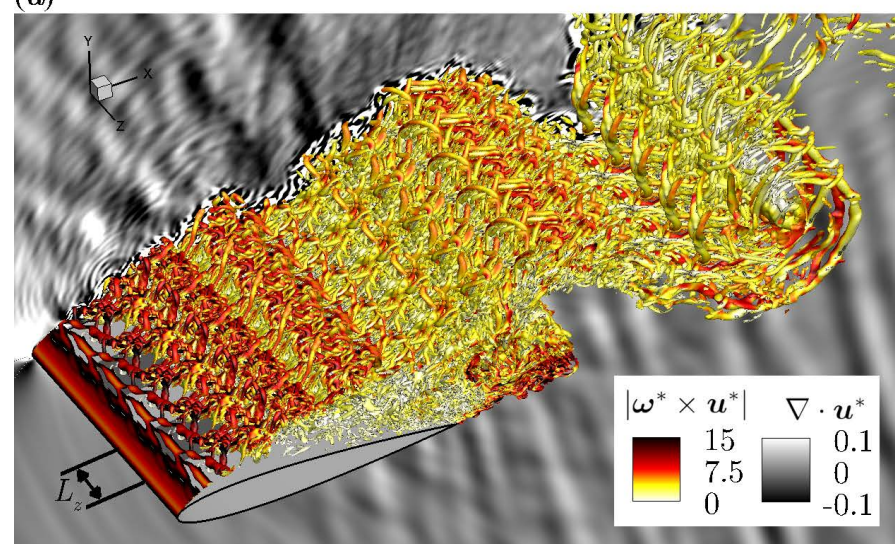

(b)

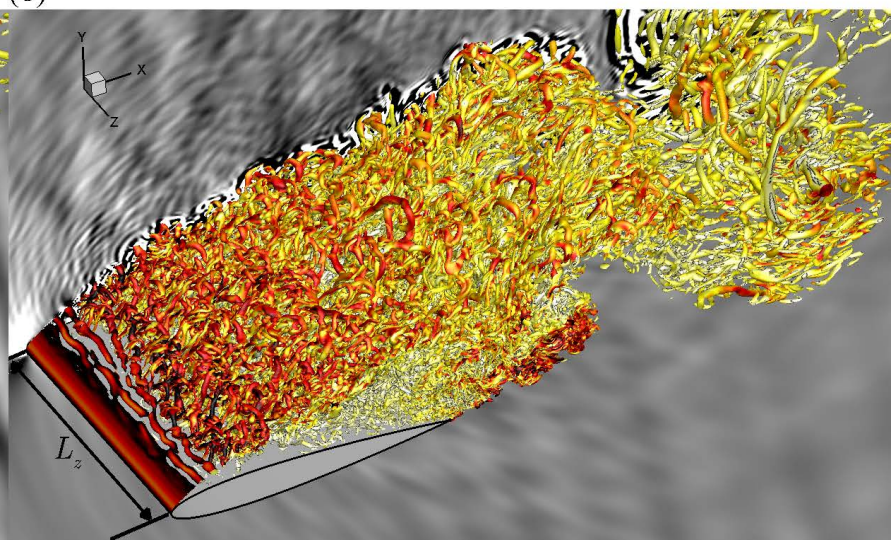

FIG. 9. Iso-surfaces of $Q$-criterion $(Q=40)$ colored by normalized Lamb vector magnitude $\left|\omega^{*} \times u^{*}\right|$. The sound field is visualized by divergence of velocity contours shown in the $x y$-plane. (a) Small domain size $L_{z}=0.2 L_{c}$ (repeated 5 times for comparison), $88.7 \mathrm{M}$ cells; Large domain size $L_{z}=L_{c}, 438.1 \mathrm{M}$ cells.

(a)

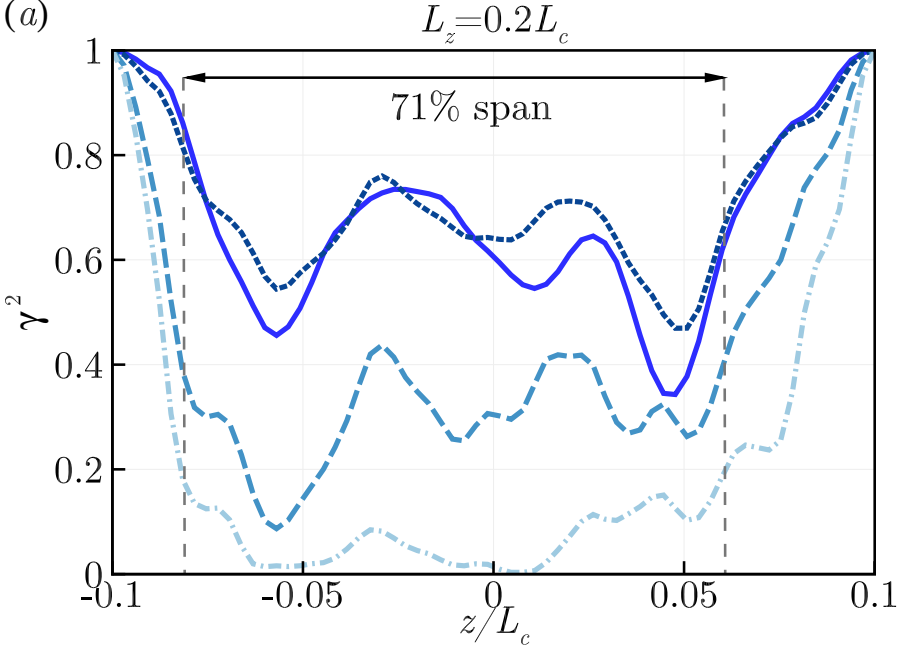

(b)

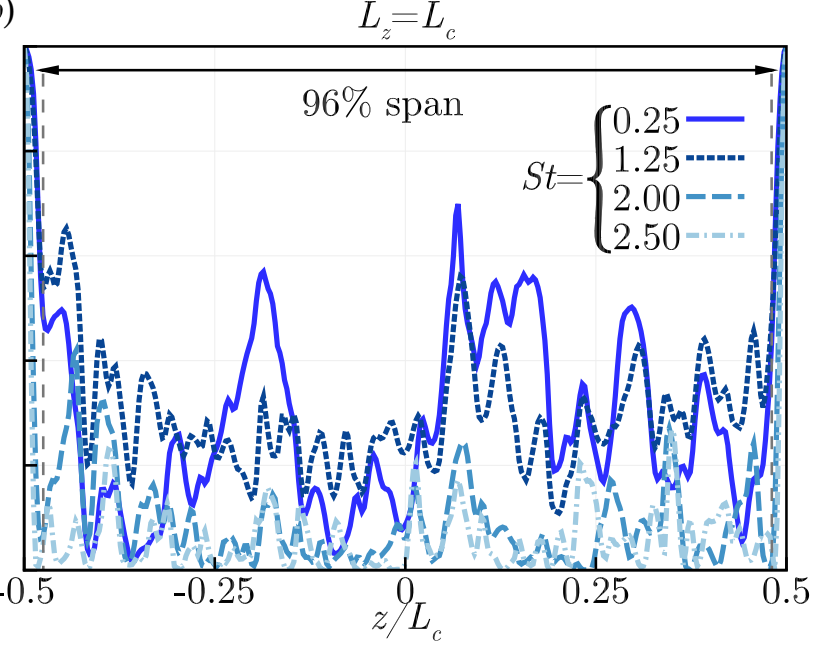

FIG. 10. Two-point coherence of pressure in the spanwise direction taken at $\mathrm{P}_{\mathrm{TE}}=\left(0.4995 \mathrm{~L}_{\mathrm{c}}, 7.26 \times 10^{-5} \mathrm{~L}_{\mathrm{c}}\right)$ (see figure 2 (c)) on the suction side of the airfoil for $\alpha=15^{\circ}$ at four Strouhal numbers $\left(S t=f L_{c} / u_{\infty}\right)$. (a) $L_{z}=0.2 L_{c}$ and $(b) L_{z}=L_{c}$.

The spanwise two point coherence of pressure on the airfoil suction side taken one point upstream of the TE is shown in figure $10(a)$ for $L_{z}=0.2 L_{c}$, and $(b)$ for $L_{z}=L_{c}$, at $\alpha=15^{\circ}$. Four frequencies are selected $S t=0.25,1.25$, 2.0 and 2.5. The figure clearly shows how the spanwise coherence for the smaller span airfoil drops at a slower rate with frequency, confirming what was suggested visually in figure 9. The coherence approaches 0 at approximately $S t=2.5$ compared to 2.0 for the larger span case. Additionally, at a lower frequency of $S t=1.25$, the coherence is around three times higher in the midspan. These findings imply that the noise generated with a smaller span will radiate more strongly than the large span case. It is also worth noting that the proportion of the span which is affected by the periodic forcing at the spanwise boundaries is much more for the small span domain size. A possible criteria for quantifying this is to consider the percentage of the span below a certain coherence level. For example, at $S t=2.5,71 \%$ of the small span obtains a coherence value less than 0.2 , this contrasts with $96 \%$ for the large spanwise domain simulation.

\section{B. Radiated noise predictions}

The predicted radiated noise time signals obtained via the FW-H solver are shown in figure $11(a)$ for $\alpha=10^{\circ}$ averaged over a narrow observer band $84^{\circ} \leq \theta \leq 96^{\circ}, 10 L_{c}$ from the airfoil mid-chord. At this angle of attack a similar maximum amplitude is achieved for all three span lengths. The PSD of acoustic pressure versus Strouhal 
$(a)$

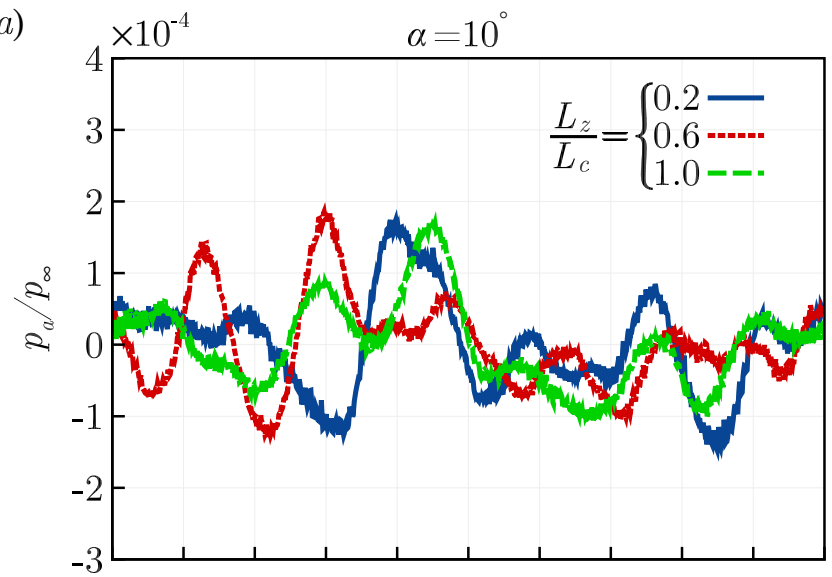

$(c)$

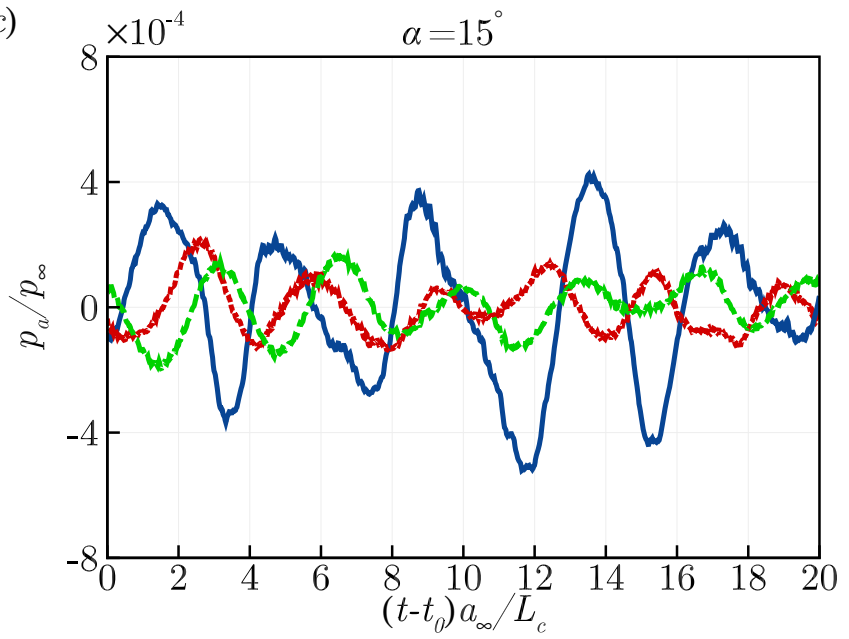

(b)

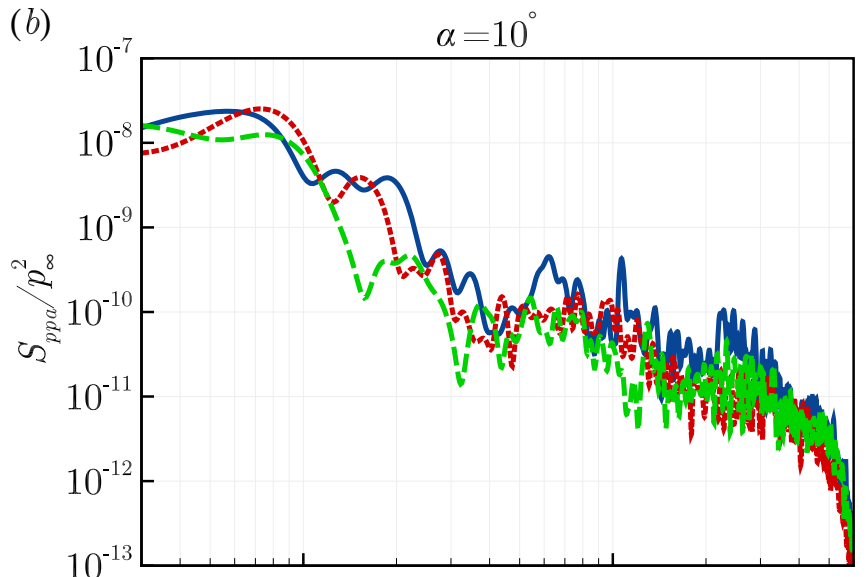

(d)

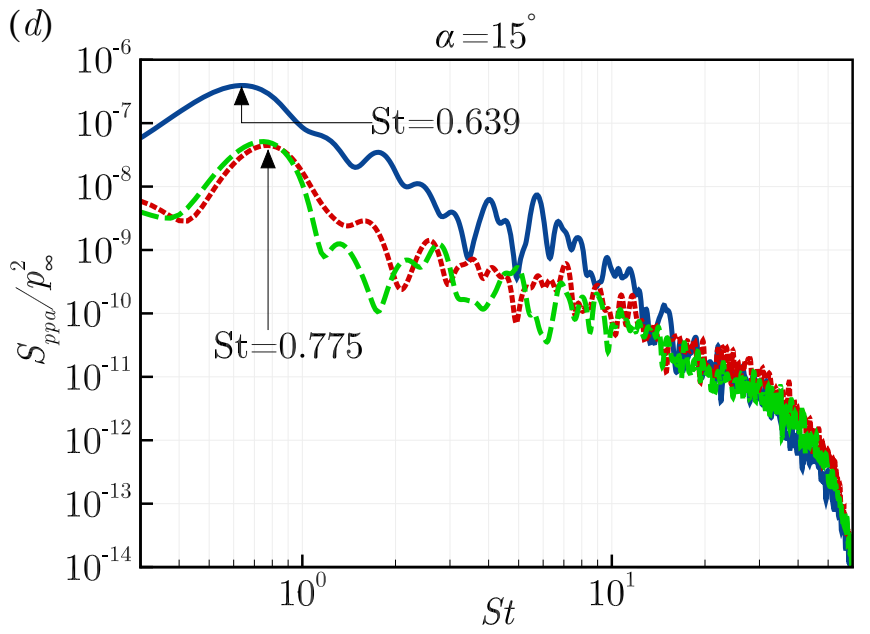

FIG. 11. Far-field acoustic data obtained by a NACA0012 airfoil at $\alpha=10^{\circ}(a) \&(b)$, and $\alpha=15^{\circ}(c) \&(d)$ for the three span lengths. $(a) \&(c)$ Acoustic pressure time signal $\left(p / p_{\infty}\right) ;(b) \&(d) \operatorname{PSD}\left(S_{p p a} / p_{\infty}^{2}\right)$. Data is averaged over a $12^{\circ}$ observer band $84^{\circ} \leq \theta \leq 96^{\circ}$.

number $\left(S t=f L_{c} / u_{\infty}\right)$ is also shown in figure $11(b)$. As implied by the time signal amplitude, the low frequency radiated noise is relatively consistent for the three span lengths. At higher frequencies, above $S t=6.0$, more significant differences emerge for the smallest span which contains moderate peaks at $S t=6.0$ and 10.8, as well as a number of smaller peaks between $22.5 \leq S t \leq 28.8$.

The times signals and spectra for $\alpha=15^{\circ}$ are shown in figure $11(c) \&(d)$ also averaged over $84^{\circ} \leq \theta \leq 96^{\circ}$. It is immediately clear how utilising a small span results in a large over-prediction of the acoustic pressure amplitude at the dominant von Kármán shedding frequency relative to the larger spanwise domain length cases. Additionally, according to the spectra in figure $11(d)$ the peak frequency is actually under-predicted for the small span airfoil, with a maximum value at $S t=0.639$ compared to $S t=0.775$ for the medium and large span cases. The PSD magnitude discrepancies between small and medium (or large) spans are around $10 \mathrm{~dB}$ or more for low-medium frequencies $(S t \leq 10)$. At higher frequencies the noise amplitude obtained by the three setups is comparable.

Sound directivity plots are now considered for the two angles of attack based on magnitude of Fourier transform of acoustic pressure $\left|P_{a}\right|$ in $1 / 3$ octave bands. Six center frequencies are included $(S t=0.8,2.0,4.0,8.0,16.0$, and $32.0)$ and contrasted for the three spanwise domain sizes at observers $\left(x_{o}, y_{o}, z_{o}\right)=\left(10 L_{c} \cos (\theta), 10 L_{c} \sin (\theta), 0\right)$. Data is included in $\Delta \theta=2^{\circ}$ intervals. Results for $\alpha=10^{\circ}$ are shown in figures $12(a)-(f)$. Despite the relatively good agreement shown by the spectra in figure $11(b)$, there is quite poor agreement for the directivity at certain frequencies, particularly at $S t=4.0$ and 8.0. At $S t=4.0$ both small and medium span airfoils predict an upstream directivity which is not observed by the large span airfoil. Similarly, at $S t=8.0$ the two smaller span cases over-predict both the upstream and downstream directed sound considerably compared to the large span case. An unexpected result is also obtained at the lowest frequency shown $(S t=0.8)$ where the small span achieves a closer match to the large span airfoil. This might suggest that the dominant low frequency mechanism at this angle are not heavily influenced by the spanwise length scale. The three remaining frequencies $(S t=2.0,16.0$ and 32.0$)$ show a more expected outcome 
(a) $S t=0.80$

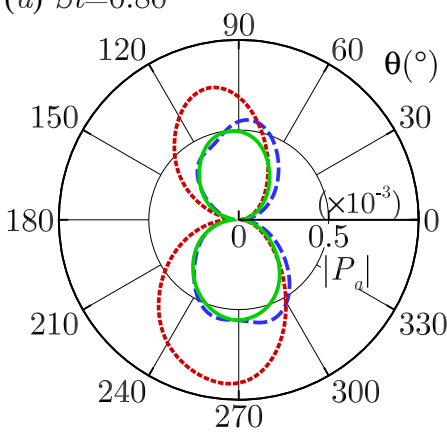

(d) 8.0

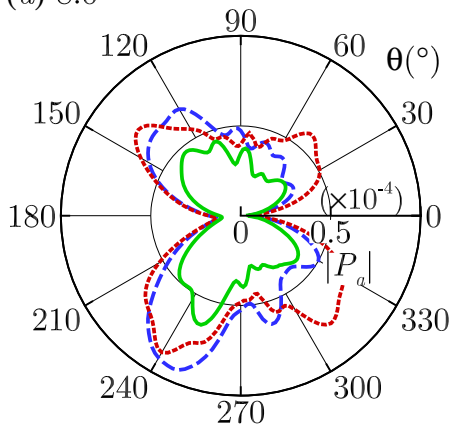

(b) 2.0

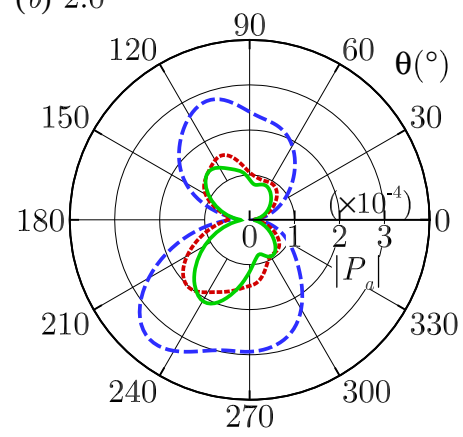

(e) 16.0

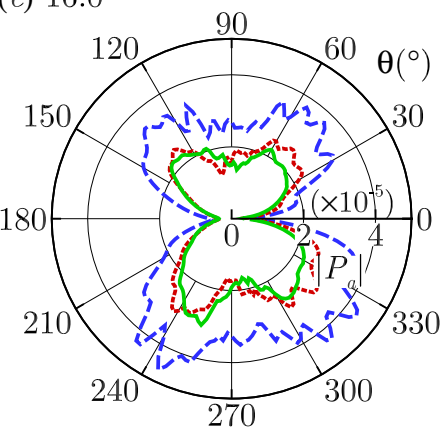

(c) 4.0

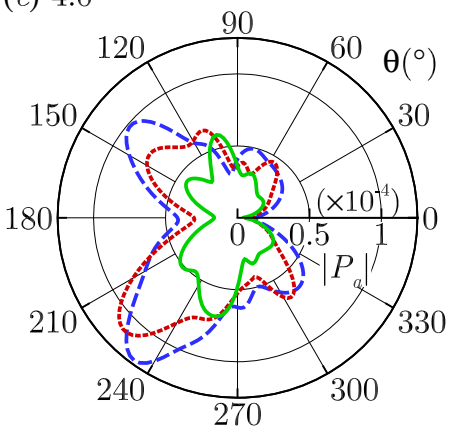

(f) 32.0

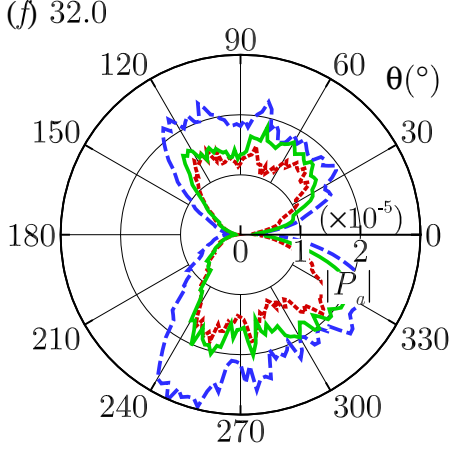

------ $L_{z}=0.2 L_{c}--------0.6 L_{c}-1.0 L_{c}$

FIG. 12. 1/3 octave band sound directivity based on magnitude of acoustic pressure Fourier transform $\left(\left|P_{a}\right|\right)$ at observers $\left(x_{o}, y_{o}, z_{o}\right)=\left(10 L_{c} \cos (\theta), 10 L_{c} \sin (\theta), 0\right)$ for the NACA0012 at $\alpha=10^{\circ}$. Results shown for six Strouhal numbers $(S t=$ $\left.f L_{c} / u_{\infty}\right)$ and the three airfoil span lengths. (a) $S t=0.8,(b) S t=2.0,(c) S t=4.0,(d) S t=8.0,(e) S t=16.0,(f) S t=32.0$.

where some level of convergence can be observed as the spanwise domain length is increased.

Equivalent directivity data is plot in figure $13(a)-(f)$ for $\alpha=15^{\circ}$. For low-medium frequencies $(a)-(d)$ the large span directivity magnitude is well predicted by the medium span setup which also in most cases agrees with the dominant radiation directions. The result may suggest that the maximum spanwise length scale does not exceed the medium span of $L_{z}=0.6 L_{c}$. For the small span airfoil, the large overestimate recognized earlier is found to persists in all observer directions for low-medium frequencies. Additionally, the small span shows some differences in directivity shape even for the lowest frequency, mainly in the lower half plane. Regarding the highest frequencies shown, $S t=16$ and 32, the directivity magnitude and pattern are comparable for all three cases. This is the expected outcome considering the associated length scales should be less than the smallest spanwise domain size.

The far-field acoustic pressure PSD averaged over all observer angles is shown in figure 14, which provides better contrast with the directivity patterns, as well as an indication of the average magnitude differences between the three cases. Generally, the differences between small span and large span are more significant for the $\alpha=15^{\circ}$ case (which is to be expected). However, it is worth noting that the higher angle converges more convincingly, while more uncertainty remains between the medium and large span results for $\alpha=10^{\circ}$. The increased sensitivity of this case may be caused by the proximity to stall onset, however this is currently unclear and should be investigated further at a later point. Two frequencies are highlighted in the figure, $S t=5 /\left(3 M_{\infty}\right)$ and $S t=5 / M_{\infty}$, corresponding to the largest acoustic wavelengths which may be captured in the spanwise direction for the medium and small span setups. They are calculated as $f=a_{\infty} / L_{z}$, or as a non-dimensional frequency $S t=L_{c} /\left(M_{\infty} L_{z}\right)$. These values indicate the lower limits below which differences might be expected between medium-large span, and small-medium span respectively. As shown by figure 14(b), this simple equation is an accurate prediction method for the frequency where differences emerge between varying span simulations when under full stall conditions. This clearly demonstrates the link between the maximum spanwise length scale and the radiated noise. Although not to the same extent, this criteria also provides some indication to where the largest differences emerge between large and smaller span lengths for the lower angle of attack, $\alpha=10^{\circ}$. In particular, significant discrepancies emerge between medium and large span airfoils below $S t=5 /\left(3 M_{\infty}\right)$. 


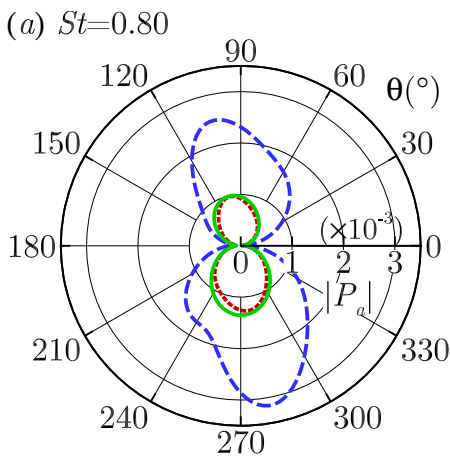

(d) 8.0

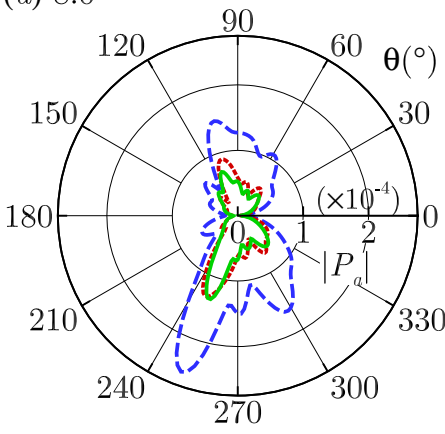

(b) 2.0

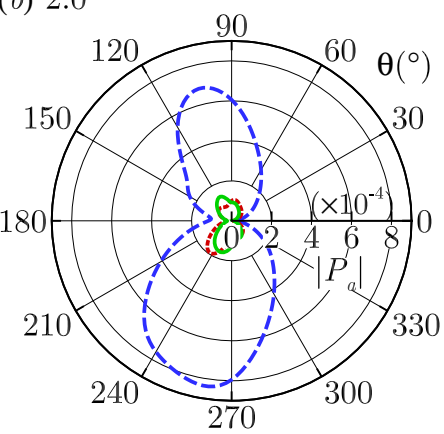

(e) 16.0

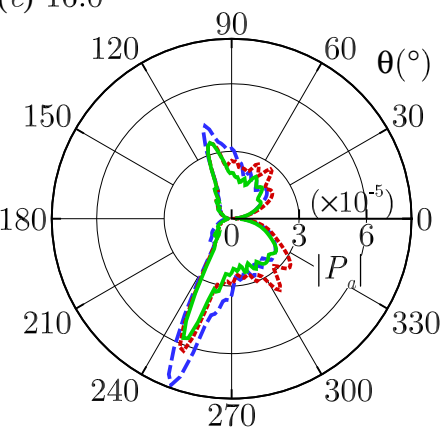

(c) 4.0

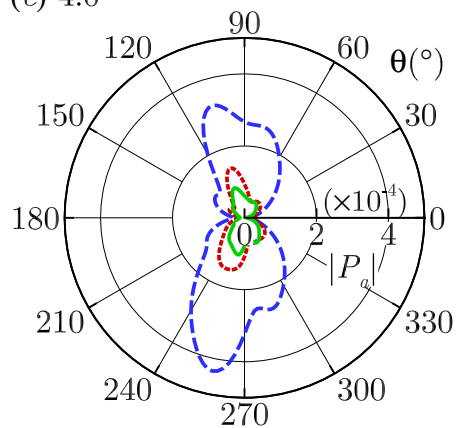

(f) 32.0

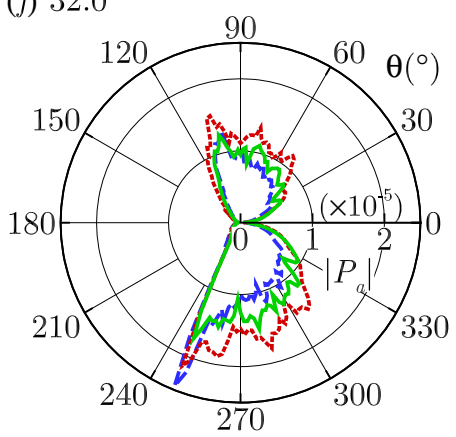

------ $L_{z}=0.2 L_{c}-\cdots-----0.6 L_{c} \longrightarrow 1.0 L_{c}$

FIG. 13. 1/3 octave band sound directivity based on magnitude of acoustic pressure Fourier transform $\left(\left|P_{a}\right|\right)$ at observers $\left(x_{o}, y_{o}, z_{o}\right)=\left(10 L_{c} \cos (\theta), 10 L_{c} \sin (\theta), 0\right)$ for the NACA0012 at $\alpha=15^{\circ}$. Results shown for six Strouhal numbers $(S t=$ $\left.f L_{c} / u_{\infty}\right)$ and the three airfoil span lengths. (a) $S t=0.8,(b) S t=2.0,(c) S t=4.0,(d) S t=8.0,(e) S t=16.0,(f) S t=32.0$.
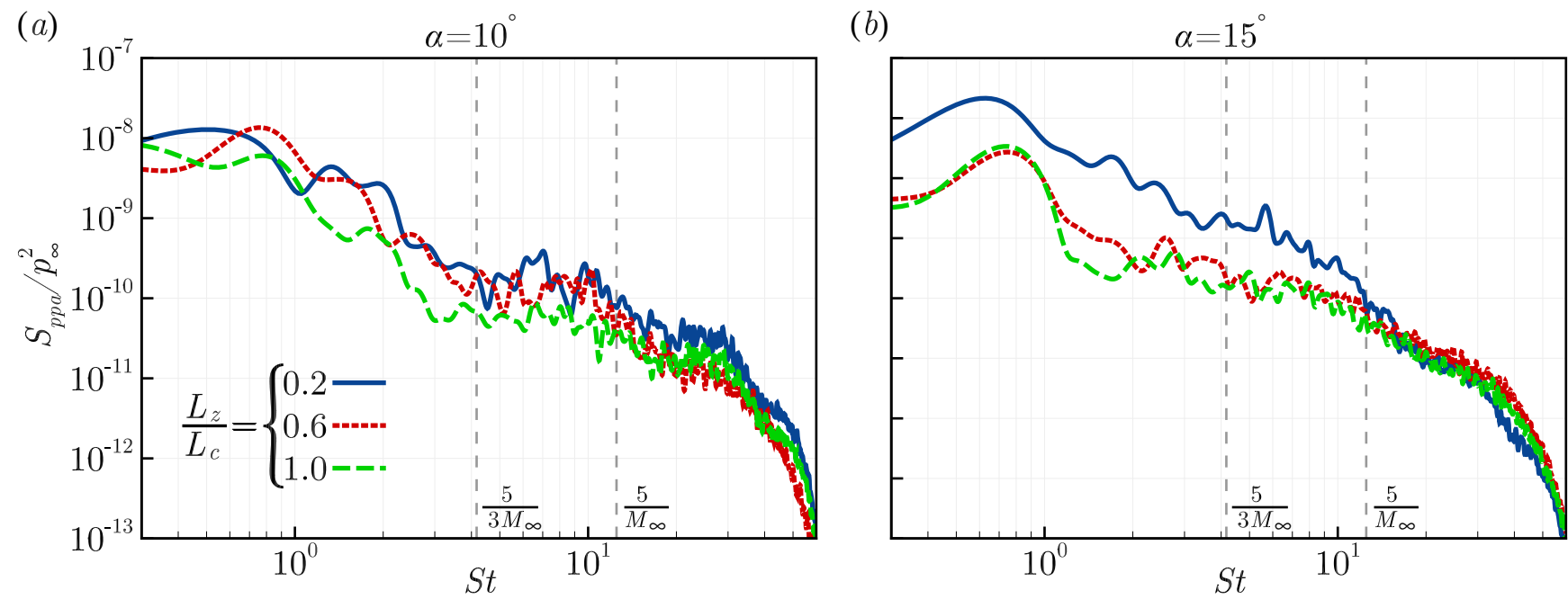

FIG. 14. PSD of far-field acoustic pressure $\left(S_{p p a} / p_{\infty}^{2}\right)$ averaged over all observer angles $0^{\circ} \leq \theta<360^{\circ}$ ten chords from the airfoil mid-chord. (a) $\alpha=10^{\circ},(b) \alpha=15^{\circ}$.

\section{Noise source characteristics}

In this section the aeroacoustic sources on the wall are investigated in order to explain some of the differences identified earlier. Figure 15 considers the Fourier transform of vertical wall pressure jump Eq. (12) plot as 2D contours over the airfoil surface for $\alpha=10^{\circ}$. Both Fourier transform magnitude (in a log scale), and the real part, are shown 
(a)

(b)

$\mathrm{TE}$

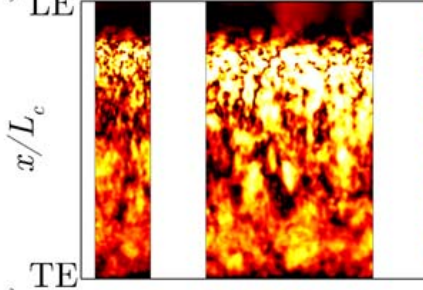

(c)

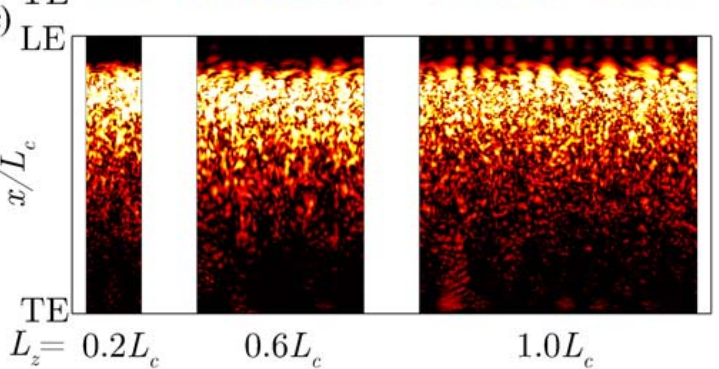

$S t=0.775$

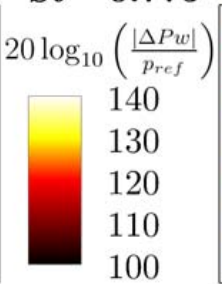

$S t=1.50$
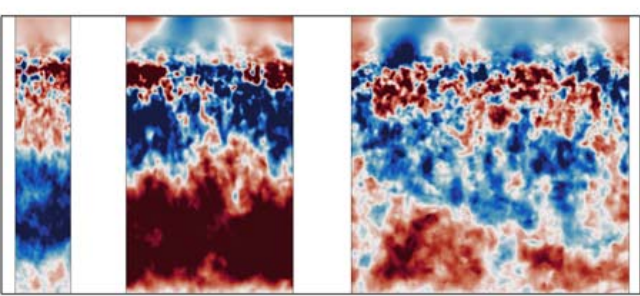

$\underline{\left|\Delta P_{w}\right| \cos (\phi)}$

$p_{\infty}$
0.02

0

$-0.02$

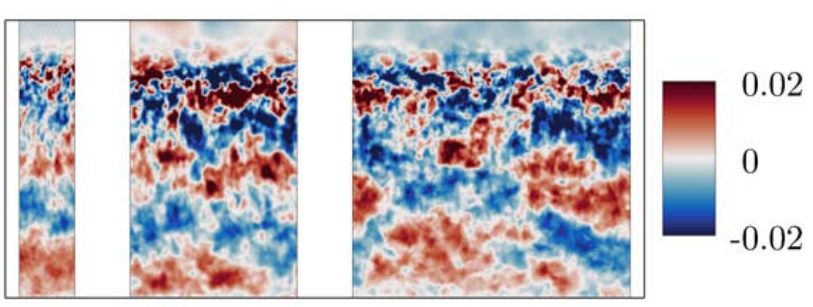

$S t=15.00$

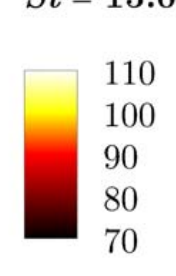

70

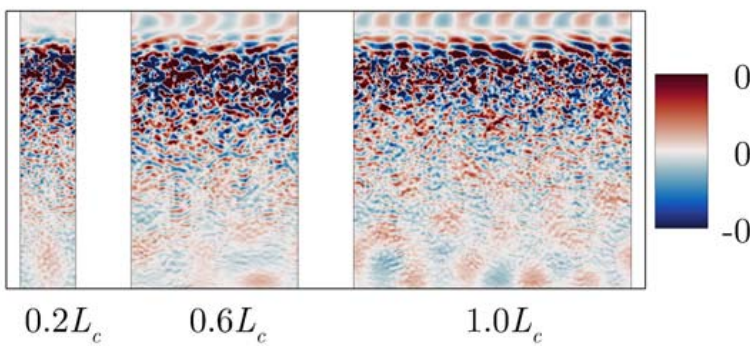

0.005

0

$-0.005$

FIG. 15. Fourier transform of wall pressure jump for $\alpha=10^{\circ}$. The left and right columns show magnitude (in logarithmic scale), and real part of Fourier transform respectively. Three frequencies are shown: $S t=0.775(a), S t=1.50(b)$, and $S t=15.00(c)$. The reference pressure used is $p_{r e f}=10^{-10} p_{\infty}$.

for $S t=0.775(a), S t=1.50(b)$, and $S t=15.00(c)$. The real part of Fourier transform is used to visualize phase changes by considering Real $\left(\Delta P_{w}\right)=\left|\Delta P_{w}\right| \cos (\phi)$, where $\phi$ is the phase angle. The imaginary part could also be used, but is not shown here for brevity.

For the lowest frequency shown $S t=0.775$, each case shows a strong source magnitude near the LE where the separation bubble is located, and a weaker source region on the aft portion of the airfoil surface. The first source region is likely due to near wall quadrupoles radiating onto the wall during transition and reattachment of separated boundary layer, while the downstream source may be due to vortex shedding in the near wake, seen as a small peak in the spectra shown earlier. One of the most noticeable differences in the source magnitude is how the reattachment point oscillates in the spanwise direction for the medium and large span airfoils. Comparatively, for the smaller span it is nearly uniform in the spanwise direction. Similar findings are made for the phase, which shows that the phase across the bubble reattachment point oscillates between positive (red), and negative (blue) only for the larger span cases. Another interesting observation is that the aft portion of airfoil has lower amplitude for the large span compared to the medium span case. Similarly this region is not entirely in-phase in the spanwise direction unlike medium and small span cases. This may explain why a peak is more pronounced at this frequency for the medium span compared to the large. At $S t=1.50$ the Fourier transform magnitude is qualitatively similar for medium and large spans with slightly reduced magnitude for the small span airfoil. Despite this, at $S t=1.50$ the radiated noise is moderately less for the large span relative to the others. Over the aft portion of the large span airfoil the phase tends to oscillate in the spanwise direction, with approximately one period captured. Relatively, the other two cases are more uniform across the span. This might explain the less efficient radiation for large span due to an increased amount of destructive interference. At the highest frequency shown $S t=15.00$, the magnitude and phase patterns are comparable with the three spanwise domain sizes. This is consistent with the far-field $\mathrm{FW}-\mathrm{H}$ prediction which shows a similar level of radiated noise for all three setups. At this frequency the highest amplitude is observed by smaller scales of turbulence appearing after transition and reattachment. Since the dominant scales are small, the phase changes considerably over a short distance, and therefore is not significantly forced by the periodic boundary condition.

The same analysis is provided for $\alpha=15^{\circ}$ in figure 16 this time for $S t=0.775(a), S t=5.00(b)$, and $S t=7.50$ $(c)$. These frequencies are selected since large difference are observed in the far-field, although for differing reasons. 

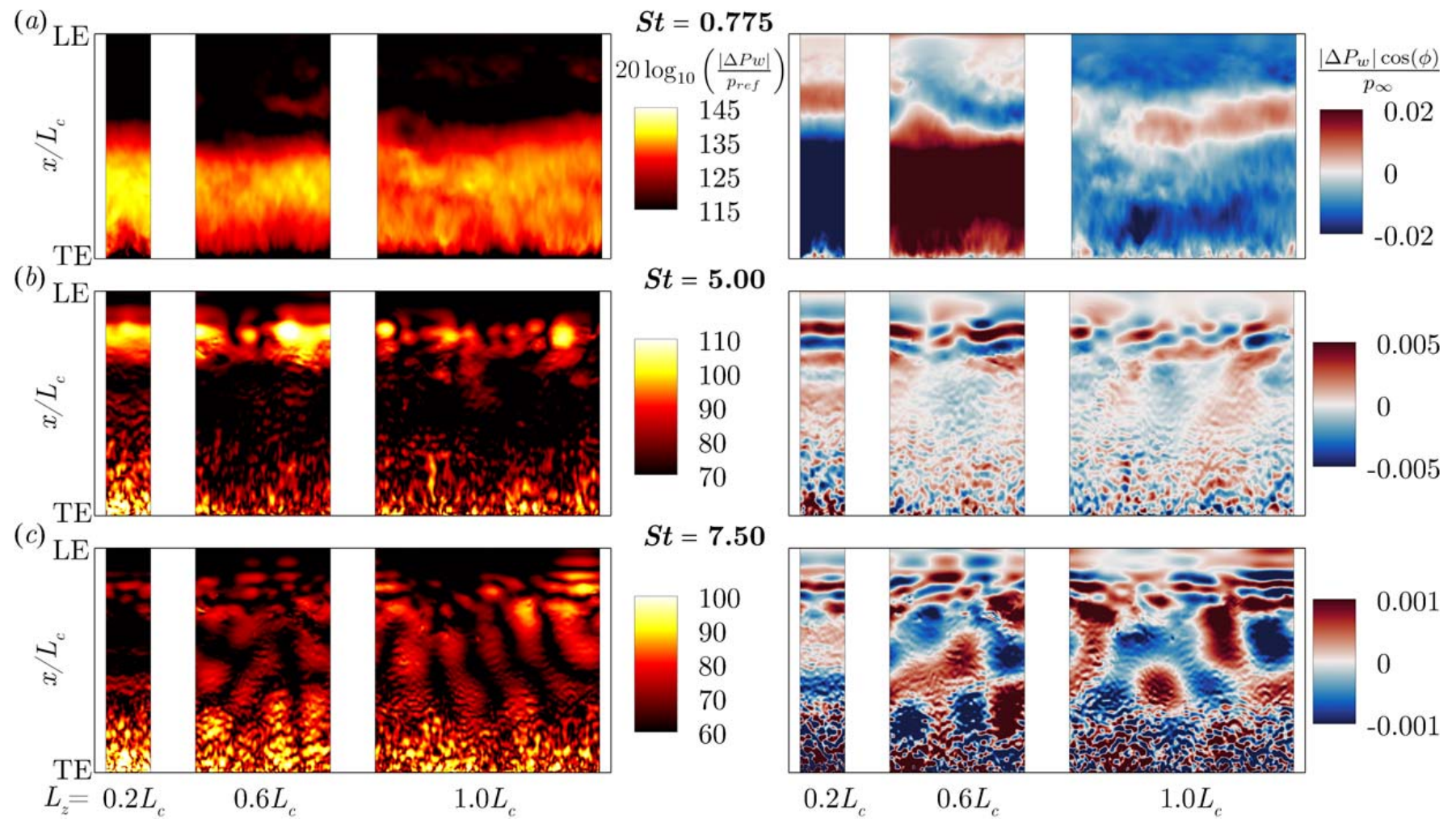

FIG. 16. Fourier transform of wall pressure jump for $\alpha=15^{\circ}$. The left and right columns show magnitude (in logarithmic scale), and real part of Fourier transform respectively. Three frequencies are shown: $S t=0.775(a), S t=5.00(b)$, and $S t=7.50(c)$. The reference pressure used is $p_{r e f}=10^{-10} p_{\infty}$.

At the lowest frequency $S t=0.775$, there is a significant reduction in source magnitude for the larger span cases, which is consistent with the observations made in the far-field. The less intense wall pressure fluctuations recorded on the surface are likely caused by the more broken up flow structures shown near the TE and in the wake. Regarding the phase relationship, each case remains approximately in-phase for the aft portion of the airfoil. However, there is slightly more variation for the largest span length case, indicating weaker constructive interference. Differences in the source magnitude are also observed at $S t=5.00$ in figure $16(b)$. There are two high strength regions, the shear layer near the LE, and turbulence close to the TE. For the medium and large span airfoils, the high strength region near the LE shows significant local hotspots, whereas for small span it is almost entirely uniform in the spanwise direction. Also, near the TE, the high strength source region extends more upstream for the small span case. At this frequency, the phase appears to play an important role for the differences observed in the far-field. This is more clearly demonstrated by figure 17, which shows the surface contours of $\left|\Delta P_{w}\right| \cos (\phi)$, repeated in span to match the FW-H integration surface $\left(L_{z}=3 L_{c}\right)$. The original computational domains are highlighted with a black border. The periodic boundary conditions for small span length have forced a more in-phase relationship over the airfoil surface in the spanwise direction. This is most apparent when comparing the high strength LE shear layer region with the larger span cases. The figure highlights that for larger spanwise domains significantly more cancellations can be expected when the surface pressure is integrated (in a FW-H solver), resulting in a lower predicted radiated noise. For this particular frequency integrating $\Delta P_{w}$ then taking the magnitude squared yields approximately a factor of three increase for the small span case. (Please note this does not correspond exactly with the far-field data due to the angle averaging approach, and other terms appearing in the FW-H equation). It is also interesting to observe that the medium span case barely captures one wavelength of oscillation in the spanwise direction at the mid-chord location. This however appears to be satisfactory for a good agreement for the radiated noise, as shown by the spectra previously. Lastly in figure 16(c), the magnitude and phase is shown for $S t=7.50$. At this frequency the high strength source region is concentrated at the TE for each case, however the maximum amplitude is notably higher for the small span (around $8 \mathrm{~dB}$ larger than the other cases). Additionally, the upstream source strength is notably weaker for the small span, which doesn't capture the same streamwise patterns observed by the other two cases. Regarding the phase, there appears to be a similar amount of in and out-of-phase regions for the three cases near the TE. However, the small span shows increased uniformity around the mid-chord. 
(a)

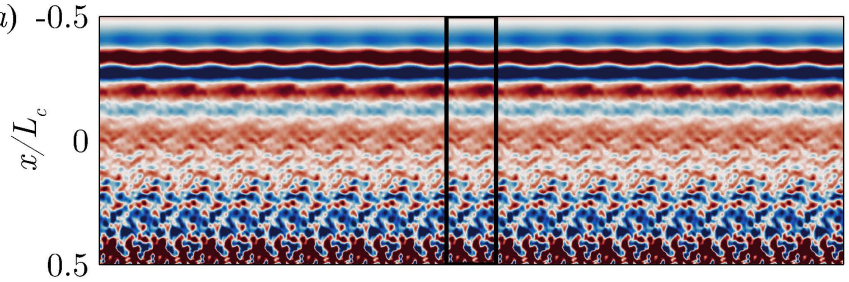

(b)

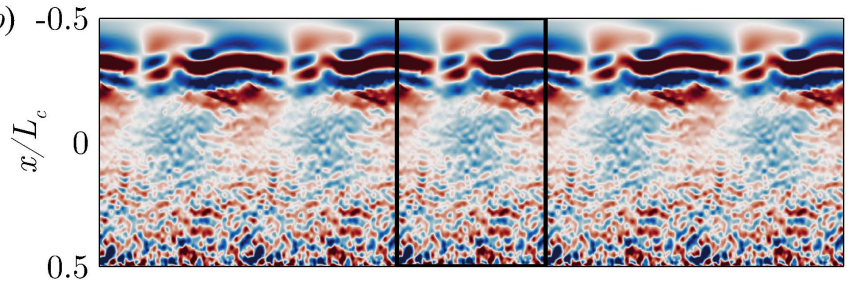

(c)

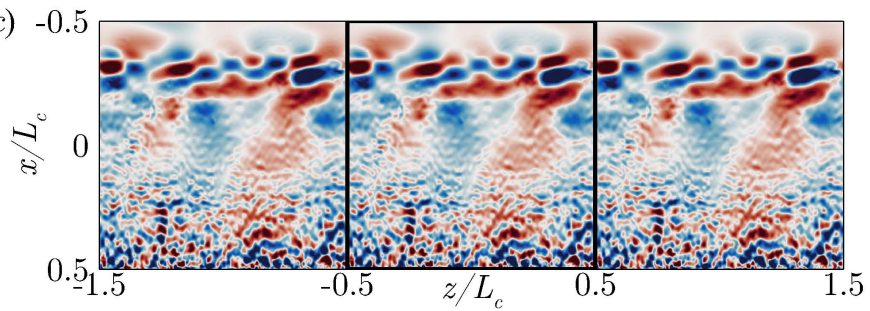

$\frac{\left|\Delta P_{w}\right| \cos (\phi)}{p_{\infty}}$

0.003

0.002

0.001

0

$-0.001$

$-0.002$

$-0.003$

FIG. 17. Contours of real part of Fourier transform of wall pressure jump $\left|\Delta P_{w}\right| \cos (\phi) / p_{\infty}$ for $S t=5.0$ and $\alpha=15^{\circ}$. Results are repeated in span until $L_{z}=3 L_{c}$ which corresponds to the FW-H domain size used. The original domain is highlighted by a black border: (a) $L_{z}=0.2 L_{c},(b) L_{z}=0.6 L_{c}$ and $(c) L_{z}=L_{c}$.

\section{CONCLUDING REMARKS}

Numerical simulations of an airfoil in near- and full-stall conditions are carried out in order to investigate how spanwise domain length influences radiated noise when spanwise periodic boundary conditions are used. The study is based on a NACA0012 airfoil at Reynolds number $R e_{\infty}=50,000$ and Mach number $M_{\infty}=0.4$. Three span lengths are tested, $20 \%, 60 \%$ and $100 \%$ of the airfoil chord length. It is well established that large spanwise domains are required to properly predict the mean aerodynamic performance of stalled airfoils in simulation approaches. In this paper it is demonstrated how aeroacoustic predictions are also greatly influenced by the choice of this parameter.

For low-medium frequencies radiated noise predictions for the smallest span exceed the larger spans by more than $10 \mathrm{~dB}$ under full stall conditions $\left(\alpha=15^{\circ}\right)$. It is also observed how small span may influence the location of important tones, for example low frequency peaks linked to large scale vortex shedding. The frequency range where agreement is reached between different span simulations is found to be strongly linked to the largest acoustic wavelength attainable in the spanwise direction. A lower limit for convergence is demonstrated as approximately $S t_{c}=L_{c} /\left(M_{\infty} L_{z}\right)$ for the full-stall simulation. The predicted noise is shown to converge rapidly as the span is increased. For near-stall conditions $\left(\alpha=10^{\circ}\right)$ discrepancies are generally smaller between small and medium span simulations. However, there is less clear convergence as the span is increased further, and some moderate differences remain between medium and large span simulations except for high frequencies.

The dipole source characteristics are also investigated in detail in order to identify the causes of the different radiated sound fields. For $\alpha=15^{\circ}$ at low frequency the spanwise periodic forcing inhibits break up of the wake and increases spanwise coherence near the TE, causing stronger unsteady loading for the small span case. For medium frequencies the noise increase for small span is further explained by considering the phase on the airfoil surface. It is shown that periodic forcing leads to more in-phase sources primarily near the leading and trailing edges. This increases the amount of constructive source interference, and consequently the radiated noise observed in the far-field.

The current work extensively studied the influence of spanwise domain length on aerodynamic and acoustic predictions due to leading edge stall, caused by the generation and subsequent bursting of a separation bubble. A logical extension to the current work would be to consider higher Reynolds number flows where trailing edge stall is more prominent. Under such conditions there is the possibility of stall cells developing which may further increase the sensitivity to spanwise domain length. This however will incur considerable extra cost, especially for large spanwise domain simulations of the order of one chord length, and therefore may be more practical through lower fidelity 
approaches.

\section{ACKNOWLEDGEMENT}

The authors gratefully acknowledge the support of the ESPRC (Engineering and Physical Sciences Research Council) under grant EP/R010900/1. We also acknowledge the high-performance computing facilities and services offered by the UK national supercomputer ARCHER, and the local IRIDIS-5 at the University of Southampton. All data supporting this study are openly available from the University of Southampton repository at http://dx.doi.org/10.5258/SOTON/D1385.

${ }^{1}$ T. F. Brooks, D. S. Pope, and M. A. Marcolini, "Airfoil self-noise and prediction," NASA reference publication 1218 (1989).

${ }^{2}$ M. R. Fink and D. A. Bailey, "Airframe noise reduction studies and clean-airframe noise investigation," NASA CR-159311 (1980).

${ }^{3}$ S. Moreau, M. Roger, and J. Christophe, "Flow features and self-noise of airfoils near stall or in stall," in 15th AIAA/CEAS Aeroacoustics Conference. AIAA Paper 2009-3198 (2009).

${ }^{4}$ A. Laratro, M. Arjomandi, B. Cazzolato, and R. Kelso, "Self-noise of NACA0012 and NACA0021 aerofoil at the onset of stall," Int. J. Aeroacoust. 16, 181-195 (2017).

${ }^{5}$ R. W. Paterson, R. K. Amiet, and C. L. Munch, "Isolated airfoil-tip vortex interaction noise," J. Aircraft 12, 34-40 (1974).

${ }^{6}$ G. Lacagnina, P. Chaitanya, T. Berk, J. H. Kim, P. Joseph, B. Ganapathisubramani, S. M. Hasheminejad, T. P. Chong, O. Stalnov, K. S. Choi, M. F. Shahab, M. Omidyeganeh, and A. Pinelli, "Mechanisms of airfoil noise near stall conditions," Phys. Rev. Fluids 4 123902 (2019).

${ }^{7}$ Y. D. Mayer, B. Zang, and M. Azarpeyvand, "Aeroacoustic characteristics of a NACA 0012 airfoil for attached and stalled flow conditions," in 25th AIAA/CEAS Aeroacoustics Conference. AIAA Paper 2019-2530 (2019).

${ }^{8}$ K. J. George and S. K. Lele, "Large eddy simulation of airfoil self-noise at high reynolds number," in 22nd AIAA/CEAS Aeroacoustics Conference. AIAA Paper 2016-2919 (2016).

${ }^{9}$ J. M. Turner and J. W. Kim, "Numerical investigation into the effect of separation and stall on aerofoil noise," in Proceedings of the 11th international symposium on turbulence and shear flow phenomena (TSFP-11), Southampton, UK (2019).

${ }^{10}$ F. M. White, Viscous Fluid Flow (McGraw-Hill, 1991).

${ }^{11}$ J. W. Kim and P. J. Morris, "Computation of subsonic inviscid flow past a cone using high-order schemes," AIAA J. 40, 1961-1968 (2002).

12 J. W. Kim and D. J. Lee, "Generalized characteristic boundary conditions for computational aeroacoustics," AIAA J. 38, 2040-2049 (2000).

${ }^{13}$ J. W. Kim, A. S. H. Lau, and N. D. Sandham, "CAA boundary conditions for airfoil noise due to high-frequency gusts," Proc. Eng. 6, 244-253 (2010).

${ }^{14}$ J. W. Kim, A. S. H. Lau, and N. D. Sandham, "Proposed boundary conditions for gust-airfoil interaction noise," AIAA J. 48, 2705-2709 (2010).

${ }^{15}$ J. W. Kim and D. J. Lee, "Generalized characteristic boundary conditions for computational aeroacoustics, part 2," AIAA J. 42, 47-55 (2004).

${ }^{16}$ J. W. Kim, "Optimised boundary compact finite difference schemes for computational aeroacoustics," J. Comput. Phys. 225, 995-1019 (2007).

${ }^{17}$ J. W. Kim, "High-order compact filters with variable cut-off wavenumber and stable boundary treatment," Comput. Fluids 39, 1168-1182 (2010).

${ }^{18}$ J. W. Kim, "Quasi-disjoint pentadiagonal matrix systems for the parallelization of compact finite-difference schemes and filters," J. Comput. Phys. 241, 168-194 (2013).

${ }^{19}$ N. J. Georgiadis, D. P. Rizzetta, and C. Fureby, "Large-eddy simulations: Current capabilities recommended practices, and future research," AIAA J. 48, 1772-1784 (2010).

${ }^{20} \mathrm{~S}$. Laizet, J. Nedić, and C. Vassilicos, "Influence of the spatial resolution on fine-scale features in DNS of turbulence generated by a single square grid," Int. J. Comput. Fluid D. 29, 286-302 (2015).

${ }^{21}$ S. Miley, "Catalog of low-Reynolds-number airfoil data for wind-turbine applications," OSTI technical report: RFP-3387 (1982), $10.2172 / 5044823$

${ }^{22}$ T. Ohtake, Y. Nakae, and T. Motohashi, "Nonlinearity of the aerodynamic characteristics of a NACA 0012 aerofoil at low Reynolds numbers," J. Jpn. Soc. Aeronaut. Space Sci. 55, 439-445 (2007).

${ }^{23}$ T. Tsuchiya, D. Numata, T. Suwa, and K. Asai, "Influence of turbulence intensity on aerodynamic characteristics of an NACA0012 at low Reynolds numbers," in 51st AIAA Aerospace Sciences Meeting. AIAA Paper 2013-0065 (2013).

${ }^{24}$ J. E. Ffowcs Williams and D. L. Hawkings, "Sound generation by turbulence and surface in arbitrary motion," Philos. Trans. R. Soc. Lond. A 264, 321-342 (1969).

${ }^{25}$ F. Farassat, "Derivations of formulations 1 and 1a of Farassat," NASA/TM-2007-214853 (2007).

${ }^{26}$ I. E. Garrick and C. E. Watkins, "A theoretical study of the effect of forward speed on the free-space sound-pressure field around propellers," NACA TN-3018 (1953).

${ }^{27}$ J. M. Turner, Aerodynamic noise from undulated leading edge aerofoils (PhD thesis, University of Southampton, 2019).

${ }^{28}$ M. E. Goldstein, Aeroacoustics (McGraw-Hill, 1976) pp. 54-59. 